\title{
Optimal bursts in turbulent channel flow
}

\author{
Mirko Farano ${ }^{1,2, \dagger}$, Stefania Cherubini ${ }^{1,2}$, Jean-Christophe Robinet ${ }^{2}$ \\ and Pietro De Palma ${ }^{1}$ \\ ${ }^{1}$ DMMM, Politecnico di Bari, Via Re David 200, 70125 Bari, Italy \\ ${ }^{2}$ DynFluid Laboratory, Arts et Métiers ParisTech, 151 Boulevard de l'Hôpital, 75013 Paris, France
}

\begin{abstract}
Bursts are recurrent, transient, highly energetic events characterized by localized variations of velocity and vorticity in turbulent wall-bounded flows. In this work, a nonlinear energy optimization strategy is employed to investigate whether the origin of such bursting events in a turbulent channel flow can be related to the presence of high-amplitude coherent structures. The results show that bursting events correspond to optimal energy flow structures embedded in the fully turbulent flow. In particular, optimal structures inducing energy peaks at short time are initially composed of highly oscillating vortices and streaks near the wall. At moderate friction Reynolds numbers, through the bursts, energy is exchanged between the streaks and packets of hairpin vortices of different sizes reaching the outer scale. Such an optimal flow configuration reproduces well the spatial spectra as well as the probability density function typical of turbulent flows, recovering the mechanism of direct-inverse energy cascade. These results represent an important step towards understanding the dynamics of turbulence at moderate Reynolds numbers and pave the way to new nonlinear techniques to manipulate and control the self-sustained turbulence dynamics.
\end{abstract}

Key words: boundary layer structure, nonlinear instability, turbulent flows

\section{Introduction}

Turbulence is a widespread complex phenomenon influencing the behaviour of a large variety of natural and engineering systems. Flow in a channel (Sano \& Tamai 2016), ocean mixing (Moum et al. 2013) and the explosion of a rotating massive star (Mösta et al. 2015) are three examples of very different phenomena characterized by turbulent dynamics involving chaotic fluctuations of the physical properties and sharing the same basic properties. The atmosphere itself, up to a hundred metres from the Earth's surface, is characterized by a turbulent, chaotic motion, deep knowledge and accurate modelling of which may have tremendous implications for improving meteorological and climatological predictions (Marusic, Mathis \& Hutchins 2010). Yet, achieving a thorough comprehension of the dynamics of wall-bounded turbulent flow remains a formidable challenge since turbulence appears in a variety of different states and patterns competing with the ordered laminar state (Barkley et al. 2015).

$†$ Email address for correspondence: m.farano@libero.it 
To determine low-order models for the onset of this chaotic motion from a laminar regime, recent studies have turned the attention to the dynamics of large-scale structures, neglecting the random small-scale motion: two main examples are the direct percolation model recently provided by Lemoult et al. (2016), and the front propagation scenario by Barkley et al. (2015), explaining the coexistence of turbulent patterns competing with the laminar state in the transitional regime.

Even when the flow reaches a fully developed turbulent regime, it remains characterized by small-scale chaotic fluctuations as well as coherent structures, i.e. fluid motions highly correlated over both space and time (Panton 2001), with characteristic wavelengths and lifetimes. From a dynamical point of view, this coherent motion carries a much larger momentum than the chaotic motion at small scales; thus, a careful characterization of such structures bears an enormous potential for modelling and controlling the self-sustained turbulence dynamics.

The first evidence of coherent motion in turbulent flows dates back to the sixties, when Kline et al. (1967) ran a series of experiments in a boundary-layer flow, observing 'surprisingly well-organized spatially and temporally dependent motions' in the form of streaks. These streaks populate the region close to the wall, the buffer layer representing the inner region, with an average spanwise spacing $\lambda_{z}^{+} \approx 100$ (where the superscript ${ }^{+}$indicates variables expressed in inner units, non-dimensionalized by the viscous length scale $\delta_{v}=v / u_{\tau}, v$ being the kinematic viscosity and $u_{\tau}$ the friction velocity).

Such streaky structures are continuously regenerated in a cycle based on the lift-up mechanism that does not depend on the outer flow, making them a robust, long-living feature of the inner layer (Hamilton, Kim \& Waleffe 1995; Waleffe 1997; Jiménez \& Pinelli 1999). Concerning this regeneration cycle, a strong consensus has been achieved in the last years about the self-sustained process theorized by Hamilton et al. (1995) and Waleffe (1997). Grounding on modal and non-modal instability analysis, these authors conjectured a cyclic process composed of the following three steps: (i) streamwise streaks originate from weak streamwise vortices, due to the inherently non-modal lift-up process; (ii) saturating nonlinearly, they become prone to secondary instability; and (iii) the consequent streak oscillations recreate streamwise vorticity by nonlinear interactions, leading back to the first step. Such a self-sustained process can explain the robustness of oscillating streaky structures observed in transitional and turbulent flows. The extension of this theory led to the discovery of self-sustained exact coherent structures (Waleffe 1998; Faisst \& Eckhardt 2003; Hof et al. 2004), which are steady, periodic or chaotic states of the phase space with few unstable directions, that populate the chaotic saddle representing wall turbulence at low Reynolds numbers (Waleffe 1998; Faisst \& Eckhardt 2003; Hof et al. 2004).

Moreover, it has been also observed (Tomkins \& Adrian 2003; Adrian 2007) that large-scale coherent structures populate the outer region of wall-bounded turbulent flows, with average spanwise length $\lambda_{z} \approx O(h)$ ( $h$ being the outer length scale, for instance the half-height of a channel flow or the boundary-layer thickness of the flow over a flat plate). These large-scale structures have the form of packets of hairpin vortices (Adrian 2007) or large-scale oscillating streaks (Tomkins \& Adrian 2003). Hwang \& Cossu (2010b) have recently shown that large-scale streaky structures remain self-sustained even when small-scale motion is artificially damped. This numerical observation has been supported by the results of a linear transient growth analysis of perturbations of a mean turbulent velocity profile, showing that large-scale streamwise streaks can be amplified by a coherent lift-up effect, without the need of smaller-scale structures to sustain this growth (Cossu, Pujals \& Depardon 2009; Pujals et al. 2009; Hwang \& Cossu 2010a). 
These results suggest a scenario based on self-sustained cycles acting at different spatial scales from the inner to the outer scale. Nevertheless, this is only a part of the complex dynamics of wall turbulence, in which inner and outer scale structures are found to influence each other, as recently shown by Hwang et al. (2016), who observed a close interaction between large-scale coherent structures and near-wall small-scale streaks. In fact, the coexistence of separate cycles at different scales does not explain the spatial and temporal intermittency of large-scale velocity fluctuations and their possible interaction with inner scale structures (Jiménez 1999; Hwang et al. 2016), neither the existence of the high-energy bursting events, inherently short lived and intermittent (Jiménez et al. 2005), recurrently observed in wall-bounded turbulent flows.

Bursting events are dynamically very important since they carry approximately $80 \%$ of the Reynolds stress production (Panton 2001), accounting for a large part of the energy transported through the flow. To characterize these vigorous events, quadrant analysis of time series data has been used, showing a sequence of Q2 $(u<0, v>0)$ events called ejections (i.e. slow fluid carried up by a positive wall-normal motion), suddenly followed by rather longer Q4 $(u>0, v<0)$ events known as sweeps (high-speed fluid pushed down towards the wall) (Bogard \& Tiederman 1986). Very recently, using a filtered and over-damped large-eddy simulation, Hwang \& Bengana (2016) observed the occurrence of bursting events for isolated attached eddies of different size, the related spanwise length scale ranging between $\lambda_{z}^{+} \approx 100$ and $\lambda_{z} \approx 1.5 h$. Therefore, these energy oscillations are inherently present in the coherent motion of a fully turbulent flow even when small-scale fluctuations are damped out.

However, despite the robustness of bursts and their main features are now well recognized, their origin is still not clear. Some authors have linked them to the secondary instability of streaks; others, to the appearance of hairpin-shaped vortical structures (Moin \& Kim 1985), which can regenerate into packets populating the outer region of the flow (Robinson 1991), in the same way as streaks populate the buffer layer. Recently, Jiménez has investigated by a linear analysis the role of the Orr mechanism in the bursting phenomenon (Jiménez 2013, 2015), showing that large-scale modes of the wall-normal velocity in a turbulent minimal channel are well described by transient Orr bursts only at short times (of order $0.15 h / u_{\tau}$ ), whereas at longer times nonlinearity becomes relevant.

This work aims at providing a thorough view of energetic structures in wallbounded turbulent flow, explaining the recurrence of bursting events as an interaction between streaky and vortical structures at different scales. The final goal is to investigate whether the formation of transient coherent structures inducing bursting events in a wall-bounded turbulent flow is governed by an energy maximization process on a suitable time scale. As bursts are short-lived and highly energetic, we use a transient growth approach in a nonlinear framework in order to unravel which kind of flow structures are able to trigger rapid events with a strong energy growth in a canonical wall-bounded turbulent flow such as the channel flow. In the same way as a linear transient growth analysis on a mean turbulent profile could explain the linear growth of streaky structures in turbulent flows (Butler \& Farrell 1993; Pujals et al. 2009), a nonlinear approach is a suitable way for studying the energetic transient events characterized by ejections and sweeps. With respect to the recent linear analysis provided by Jiménez (2013, 2015), we take into account nonlinear interactions since the time horizon of the optimization analysis is larger than the linear time interval limit indicated by Jiménez (2015).

The paper is organized as follows: in the second section we define the problem and describe the employed numerical methodology; in the third section the main features 
of the nonlinear optimal coherent structures and their time evolution are discussed; and in the last section, some conclusions are provided. The details of the derivation of the governing equations are provided in the Appendix.

\section{Problem formulation}

This work provides the analysis of turbulent channel flow at friction Reynolds number $R e_{\tau}=u_{\tau} h / v=180 ; u_{\tau}, h$, and $v$ being the friction velocity, the half-height of the channel and the kinematic viscosity, respectively. Since two scalings of the variables are employed, variables expressed in inner units (normalized using $u_{\tau}$ and viscous length scale, $\delta_{v}=v / u_{\tau}$ ) are indicated with the superscript ${ }^{+}$, whereas variables without any superscript are scaled in outer units (normalized using the centreline velocity $U_{c}$ and $h$ ). Incompressible flow is computed by solving the Navier-Stokes equations (NS) in a box having streamwise, wall-normal and spanwise dimensions equal to $l_{x}=4 \pi, l_{y}=2, l_{z}=2 \pi$, respectively. No-slip boundary conditions for the three velocity components are imposed at the walls, whereas periodicity is prescribed in the streamwise and spanwise directions.

The nonlinear evolution of perturbations of the mean turbulent velocity profile is computed by solving the following system of equations $(\widetilde{N S})$ :

$$
\left.\begin{array}{c}
\frac{\partial \tilde{\boldsymbol{u}}}{\partial t}=-\tilde{\boldsymbol{u}} \cdot \nabla \tilde{\boldsymbol{u}}-\tilde{\boldsymbol{u}} \cdot \nabla \boldsymbol{U}-\boldsymbol{U} \cdot \nabla \tilde{\boldsymbol{u}}-\nabla \tilde{p}+\frac{1}{R e} \nabla^{2} \widetilde{\boldsymbol{u}}+\nabla \cdot \boldsymbol{\tau}, \\
\nabla \cdot \tilde{\boldsymbol{u}}=0,
\end{array}\right\}
$$

where $\widetilde{\boldsymbol{u}}=(\widetilde{u}, \widetilde{v}, \widetilde{w})^{\mathrm{T}}$ and $\tilde{p}$ represent the velocity and pressure perturbations, respectively, which are composed of a coherent and a fluctuating part; $\boldsymbol{U}$ is the mean turbulent flow velocity profile; $\boldsymbol{\tau}$ is the Reynolds stress tensor forcing the mean turbulent velocity profile (see (A 2) in the Appendix); and $R e=U_{c} h / \nu$. The Reynolds stress tensor is computed a priori by direct numerical simulation (DNS) of the fully turbulent flow. Details of the derivation of (2.1) are provided in the Appendix.

Using (2.1), we look for perturbations capable of inducing a peak of kinetic energy in a finite time $T$. Thus, we maximize the kinetic energy growth at time $T, G(T)=$ $E(T) / E(0)$, where

$$
E(t)=\{\tilde{\boldsymbol{u}}(t), \tilde{\boldsymbol{u}}(t)\}=\int_{V}\left(\widetilde{u}^{2}+\widetilde{v}^{2}+\widetilde{w}^{2}\right)(t) \mathrm{d} V,
$$

and $V$ is the volume of the computational domain. The energy gain $G(T)$ is maximized using a Lagrange multiplier approach, the initial energy $E_{0}$, equations (2.1) and the incompressibility condition being imposed as constraints using the Lagrange multipliers or adjoint variables $\left(\widetilde{\boldsymbol{u}}^{\dagger}, p^{\dagger}, \lambda\right)$, as follows:

$$
\mathfrak{L}=\frac{E(T)}{E(0)}-\int_{0}^{T}\left\{\widetilde{\boldsymbol{u}}^{\dagger}, \widetilde{N S}\right\} \mathrm{d} t-\int_{0}^{T}\left\{\widetilde{p}^{\dagger},(\nabla \cdot \widetilde{\boldsymbol{u}})\right\} \mathrm{d} t-\lambda\left(\frac{E(0)}{E_{0}}-1\right) .
$$

Deriving the functional $\mathfrak{L}$ with respect to the variables $\widetilde{\boldsymbol{u}}, \tilde{p}$, one obtains the following adjoint equations:

$$
\left.\begin{array}{c}
\frac{\partial \widetilde{\boldsymbol{u}}^{\dagger}}{\partial t}=\widetilde{\boldsymbol{u}}^{\dagger} \cdot(\nabla \boldsymbol{U})^{\mathrm{T}}-\boldsymbol{U} \cdot \nabla \widetilde{\boldsymbol{u}}^{\dagger}-\nabla p^{\dagger}-\frac{1}{R e} \nabla^{2} \widetilde{\boldsymbol{u}}^{\dagger}+\widetilde{\boldsymbol{u}}^{\dagger} \cdot(\nabla \widetilde{\boldsymbol{u}})^{\mathrm{T}}-\widetilde{\boldsymbol{u}} \cdot \nabla \widetilde{\boldsymbol{u}}^{\dagger}, \\
\nabla \cdot \tilde{\boldsymbol{u}}^{\dagger}=0,
\end{array}\right\}
$$


as well as the gradient of $\mathfrak{L}$ with respect to the initial perturbation, which has to be nullified in order to maximize the given $\mathfrak{L}$. Following previous works focusing on nonlinear optimal perturbations of laminar base flows (see Cherubini et al. 2010; Pringle, Willis \& Kerswell 2012; Rabin, Caulfield \& Kerswell 2012; Cherubini \& De Palma 2013; Duguet et al. 2013; Cherubini, De Palma \& Robinet 2015), the optimization problem is solved by direct-adjoint iterations coupled with a gradient rotation algorithm (Foures, Caulfield \& Schmid 2013; Farano et al. 2016). Computations are performed using the spectral-element code Nek5000 (Fischer, Lottes \& Kerkemeir 2008), with Legendre polynomial reconstruction of degree 7 and second-order accurate Runge-Kutta time integration (Deville, Fischer \& Mund 2002). The iterative procedure is stopped when the relative variation between two successive direct-adjoint loops, $e=\left(G^{n}-G^{n-1}\right) / G^{n}$ is smaller than $10^{-7}, n$ being the iteration number. Depending on the selected target time, 40-80 direct-adjoint iterations are needed for reaching convergence for one set of parameters, each optimization needing an overall computational time of 100.000-800.000 hours on an IBM cluster Intel ES 4650 .

The flow parameters are chosen according to the DNS of turbulent channel flow performed by Kim, Moin \& Moser (1987) for $R e_{\tau}=180$. The computational domain is discretized by 24,20 and 20 elements in the $x, y$ and $z$ spatial directions, respectively, obtaining a total number of grid points equal to $192 \times 160 \times 160$. For this setting we obtain approximately the values of $\Delta x^{+} \approx 12, \Delta z^{+} \approx 7, \Delta y_{\max }^{+} \approx 4.4$ and $\Delta y_{\min }^{+} \approx 0.05$, similar to those used by Kim et al. (1987). DNS has been run for approximately 13 time units $\left(t u_{\tau} / h\right)$ in order to evaluate the mean flow and the Reynolds stress tensor. The fully turbulent flow obtained by DNS has been validated by comparing the mean flow and the Reynolds shear stress with the results of Kim et al. (1987), finding a very good agreement (see the Appendix for details). Furthermore, the direct-adjoint routine has been validated by computing linear optimal perturbations following the approach by Pujals et al. (2009), who performed a local stability analysis by considering a monochromatic sinusoidal coherent perturbation in $x$ and z. Although we perform a global analysis, where $\tilde{\boldsymbol{u}}$ depends on the three spatial coordinates without any constraint, in the linear limit we have been able to reproduce the maximum energy amplification, the associated time as well as the shape of the optimal perturbations with their wavelength, found by a local linear optimization using the approach of Pujals et al. (2009). This result validates our direct-adjoint procedure at least in the linear limit. Finally, the nonlinear optimization approach has been validated in the laminar case with the results of Farano et al. (2015).

\section{Results}

\subsection{Optimal perturbations}

Nonlinear optimal structures have been computed for $\operatorname{Re}_{\tau}=180$ and for different target times, $T$, which is a crucial independent parameter for the optimization procedure. For such a moderate value of the Reynolds number, there is not a clear spatial scale separation among the flow structures. However, structures with different scales and dynamics may be found in the inner and outer region (Chen et al. 2014). The lifetime of coherent structures populating the flow can be employed as the target time to select the scale of the structures to be optimized. Butler \& Farrell (1993) have chosen as representative of the time scale of coherent structures the eddy turnover time defined as the ratio between the turbulent kinetic energy and the dissipation rate, $k / \epsilon$. In particular, they consider the value of the eddy turnover time at $y^{+} \approx 20$ (resulting 
(a)

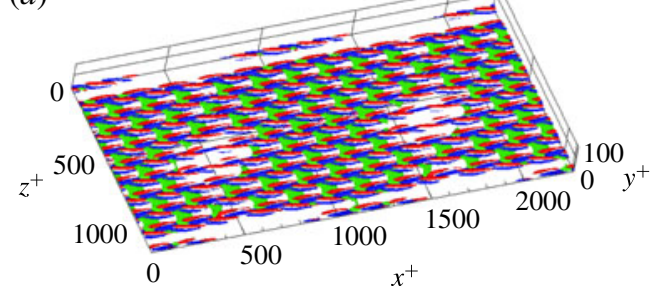

(b)

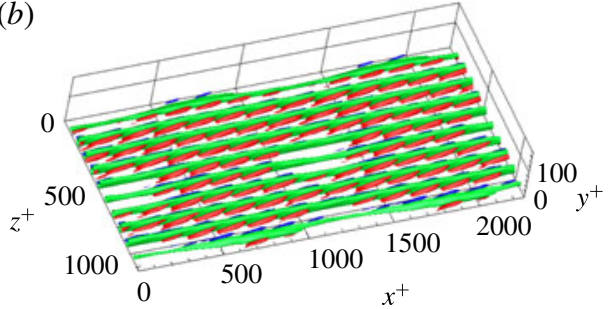

FIgURE 1. (Colour online) Shape of the optimal perturbation for $T_{i n}^{+}=80$ and $E_{0}=10^{-2}$ at $(a) t^{+}=0$ and $(b) t^{+}=T_{i n}^{+}$: isosurface of negative streamwise velocity (green, $(a) \tilde{u}=$ -0.025 , (b) $\tilde{u}=-0.18$ ) and $Q$-criterion ( $(a) Q=10^{-6}$, (b) $Q=2 \times 10^{-6}$ ) coloured by the value of the streamwise vorticity (positive blue, negative red).

in $T^{+}=80$ for $R e_{\tau}=180$ ), finding optimal small-amplitude disturbances having the shape of low- and high-speed streaks with the inner typical spanwise spacing $\lambda_{z}^{+}=110$. Here, we employ the same criterion, choosing the inner optimization time $T_{i n}^{+}=80$, $\left(T_{i n}=8.16\right)$ approximately corresponding to one eddy turnover time evaluated in the buffer layer at $y^{+}=19$ (Butler \& Farrell 1993); and the outer optimization time $T_{\text {out }}^{+}=305\left(T_{\text {out }}=31.12\right)$, corresponding approximately to one eddy turnover time at the centreline of the channel, consistent with that used by Pujals et al. (2009) for higher Reynolds numbers.

\subsubsection{Optimal perturbations at the inner time scale}

The resulting optimal finite-amplitude disturbance obtained for $T_{i n}^{+}$and $E_{0}=10^{-2}$ is shown in figure 1 at $t^{+}=0(a)$ and $t^{+}=T_{i n}^{+}(b)$. The initial optimal perturbation consists of alternated inclined streamwise vortices (red and blue), flanking localized regions of streamwise velocity strong defects (green). Whereas, at $T_{i n}^{+}$, the optimal disturbance consists of highly modulated streaks having a typical spanwise spacing of $\lambda_{z}^{+} \approx 113$, surrounded by positive and negative streamwise vortices, with a spanwise spacing of $\lambda_{z}^{+} \approx 56$; this is a typical value recovered for vortex spacing in turbulent channel flow (Panton 2001). These nonlinear optimal streaks and vortices appear much more similar to the oscillating coherent streaky structures observed in turbulent flows than the idealized linear optimal perturbations presenting a perfect streamwise alignment (Butler \& Farrell 1993; Pujals et al. 2009). Moreover, they are localized in space in a spot-like fashion, instead of occupying homogeneously the whole computational domain like the linear optimal ones. These nonlinear optimal structures well represent the self-sustained turbulence wall cycle: linearly growing streaks saturate and oscillate due to secondary instability, regenerating new localized quasi-streamwise vortices by nonlinear coupling. Linear optimizations are able to describe only the first step of this cycle, whereas a nonlinear approach can capture all of the elements of the cycle. Notice that a very similar optimal structure has been recovered for smaller target times, made by oscillating coherent streaks and vortices at the walls, having smaller wavelength (for instance, $\lambda_{z}^{+} \approx 65$ for $T^{+}=21.94$ ) but a very similar spot-like spatial localization. However, as already known (Jiménez \& Moin 1991), these streaks are not self-sustained. In fact, we have verified that the time evolution of these optimal small structures beyond the target time leads to their decay. Concerning the influence of $E_{0}$, we have observed that the optimal disturbances keep a similar structure as long as the initial energy is sufficiently high to trigger nonlinear effects. 
(a)

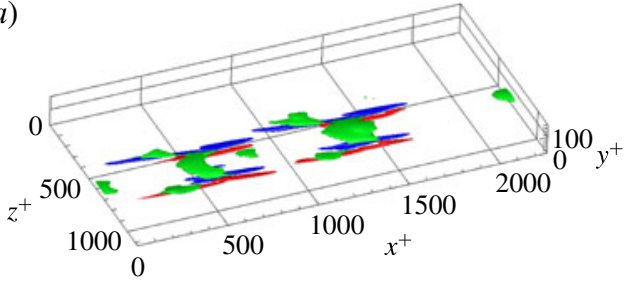

(b)

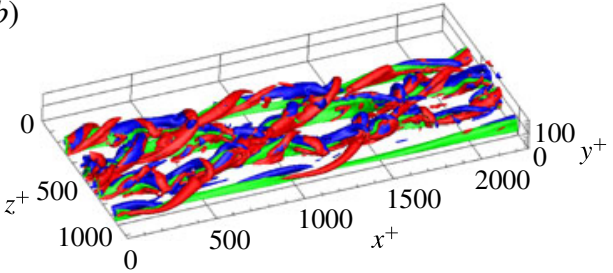

FIGURE 2. (Colour online) Shape of the optimal perturbation for $T_{\text {out }}^{+}=305$ and $E_{0}=$ $10^{-2}$ at $(a) t^{+}=0$ and $(b) t^{+}=T_{\text {out }}^{+}$: isosurface of negative streamwise velocity (green, $(a)$ $\tilde{u}=-0.016,(b) \tilde{u}=-0.3)$ and $Q$-criterion $((a) Q=0.045,(b) Q=0.15)$ coloured by the value of the streamwise vorticity (positive blue, negative red).

(a)

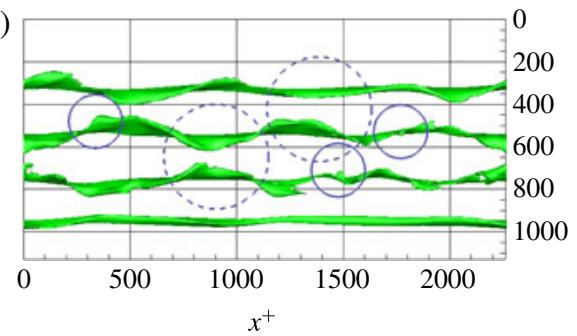

(b)

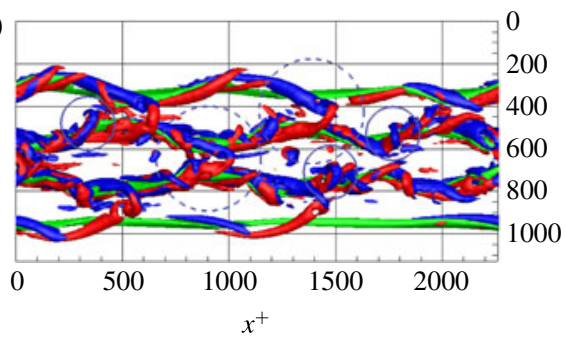

FIgURE 3. (Colour online) Shape of the optimal perturbation for $T_{\text {out }}^{+}=305$ and $E_{0}=10^{-2}$ at $t^{+}=T_{\text {out }}^{+}$: isosurface of negative streamwise velocity (green) $(a, b)$; isosurface of $Q$-criterion coloured by contours of streamwise vorticity (positive blue, negative red) (b). The isosurface values are the same as in figure 2(b). Small solid circles indicate small hairpin vortices, big dashed circles indicate big hairpin vortices.

The results obtained for the inner time scale are in good agreement with the wellassessed streaky structures observed near the wall by several authors by experimental and numerical techniques (Kline et al. 1967; Panton 2001); therefore, such results can be considered a successful validation of the proposed approach.

\subsubsection{Optimal perturbations at the outer time scale}

Increasing the target time to the outer time scale $T_{\text {out }}^{+}$, the optimization algorithm provides a different flow structure, as shown in figure 2 , at $t^{+}=0(a)$ and $t^{+}=T_{\text {out }}^{+}$ (b). The initial optimal perturbation is strongly localized in space and is characterized by alternated streamwise vortices (red and blue) near the wall and localized patches of streamwise velocity perturbations in the outer region. At $t^{+}=T_{\text {out }}^{+}$this initial perturbation turns into a much more complex structure, mostly composed of packets of hairpin vortices on top of highly oscillating streamwise streaks. In particular, strong vortical structures are observed at two different scales. The small-scale structures are not symmetric and have spanwise length $\lambda_{z}^{+} \approx 100$ (consistent with the observations of Zhou et al. (1999)). They are placed on top of the low-speed streaks, apparently as a result of their sinuous instability, as shown in the solid circles in figure 3 . On the other hand, the largest vortical structures in the dashed circles have a clear symmetric hairpin shape, with typical wavelengths $\lambda_{z} \approx 2 h$ and $\lambda_{x} \approx 2.5 h$, consistent with the observations of turbulent bulges and packets of hairpin of length $\approx 2 h$ (Adrian 2007) and with the dimensions of the largest attached eddy computed 


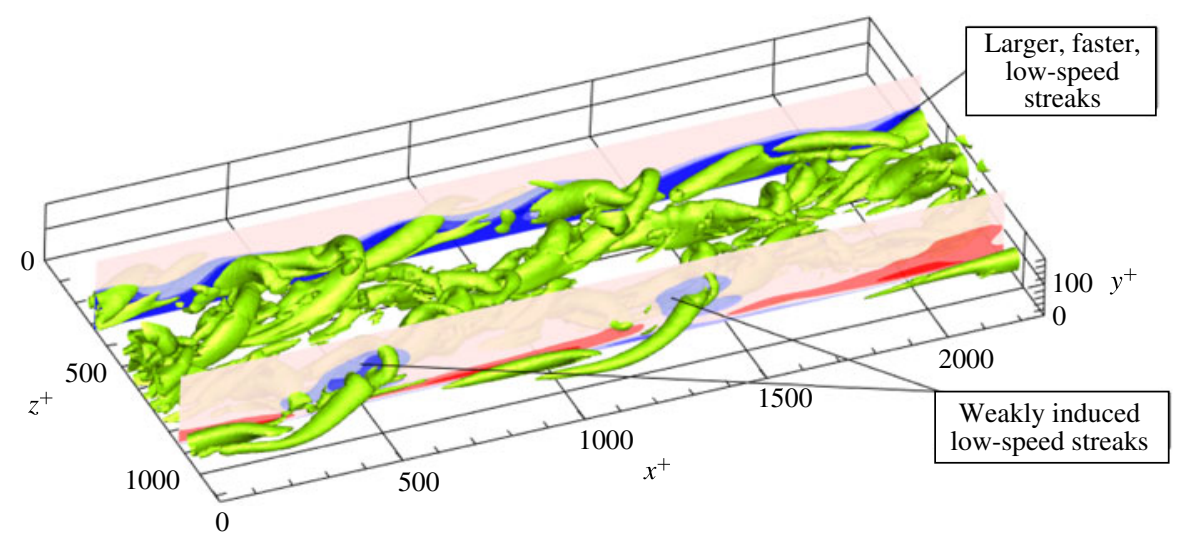

FIgURE 4. (Colour online) Outer optimal perturbation obtained for $T_{\text {out }}^{+}=305$ and $E_{0}=10^{-2}$ : isosurfaces of the $Q$-criterion (green) and isocontours of streamwise velocity (blue negative, red positive) on the planes $z^{+}=860$ and $z^{+}=320$. The isosurfaces values are the same as in figure $2(b)$.

using large-eddy simulation by Hwang \& Bengana (2016). As one can observe in figure 3, these hairpin vortices originate from the merging of the streamwise vortical structures flanking two distinct low-speed streaks (Adrian 2007), which are modulated quasi-symmetrically with respect to a streamwise axis passing between them. Large-scale low-speed streaks, with $\lambda_{z} \approx 2.2 h$ and $\lambda_{x} \approx 5 h$ are also induced between the legs of these large hairpin structures; these streaks can be observed in figure 4 in a $z$-constant plane passing through the head of two large hairpin vortices (blue contours in the plane at $z^{+}=860$ for low-speed large-scale streaks). However, the streaks with higher intensity are those close to the wall, as shown by the blue contours in the $z^{+}=320$ plane. These features recall those found for packets of hairpin vortices described by Adrian (2007), who observed that the larger the packets, the weaker the backward-induced flow, due to the larger distance of the side vortices from the centre of the hairpin loop. The same author also conjectured that the passage of hairpin packets can explain the occurrence of multiple second- and fourth-quadrant events typical of turbulent bursts.

\subsubsection{Probability density function analysis}

Thus, we wonder whether this optimal perturbation characterized by a very complex shape, optimizing the energy at the outer spatial scale, might be a possible candidate for explaining the onset of transient recurrent bursts on top of the long-living oscillating streaks. To investigate whether ejections and sweeps could characterize the dynamics of the nonlinear optimal structure, indicating a strong correlation with bursting events, we have computed the probability density function (PDF) of the streamwise and wall-normal velocity disturbance at different wall-normal positions for the optimal perturbation at $t^{+}=T_{\text {out }}^{+}$. Figure 5 shows, for $y^{+}=10,50,100(a, c, e)$, that the PDF is concentrated in the second and fourth quadrants of the $\tilde{u}-\tilde{v}$ plane, indicating the prevalence of ejection and sweep events, exactly as in a strong bursting event. Going from the buffer to the outer region, the strongest contribution to the Reynolds stress slightly moves towards the sweeps region, suggesting a mechanism of energy redistribution from the outer to the wall region by means of sweep events (Jiménez 1999). This PDF has been compared with that extracted from the DNS of the 


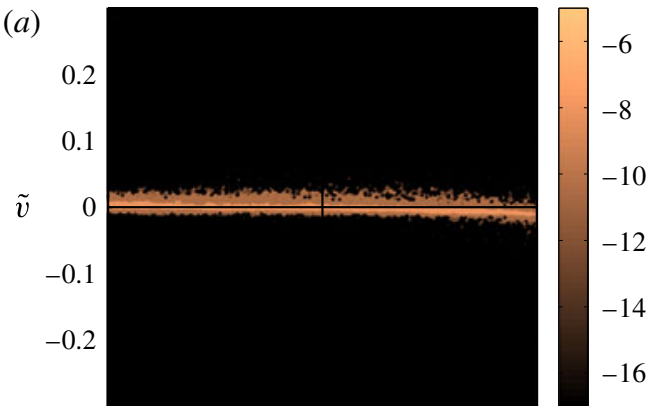

$\begin{array}{lllll}-0.2 & -0.1 & 0 & 0.1 & 0.2\end{array}$

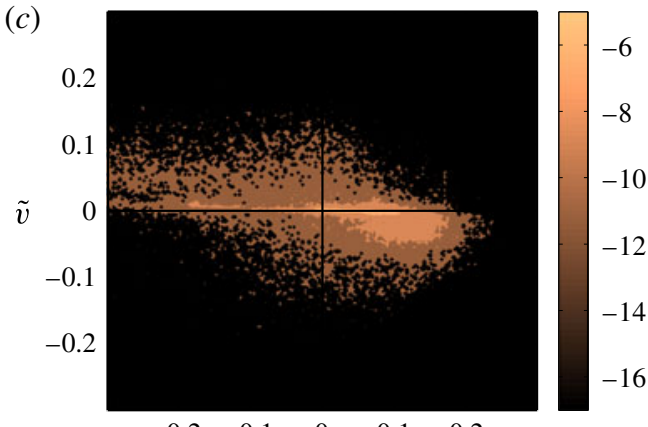

$\begin{array}{lllll}-0.2 & -0.1 & 0 & 0.1 & 0.2\end{array}$

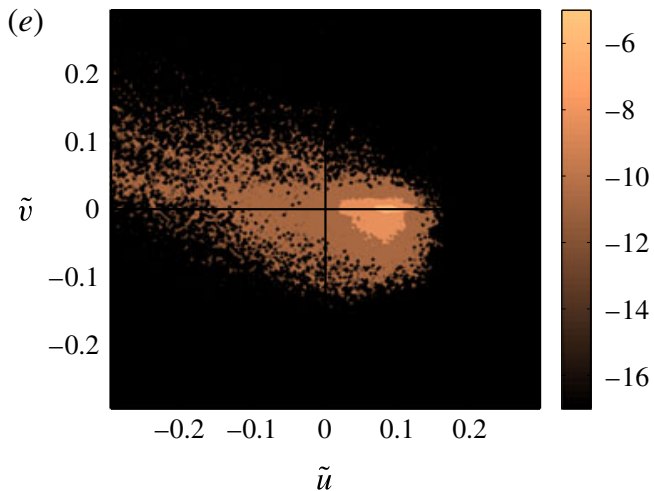

(b)

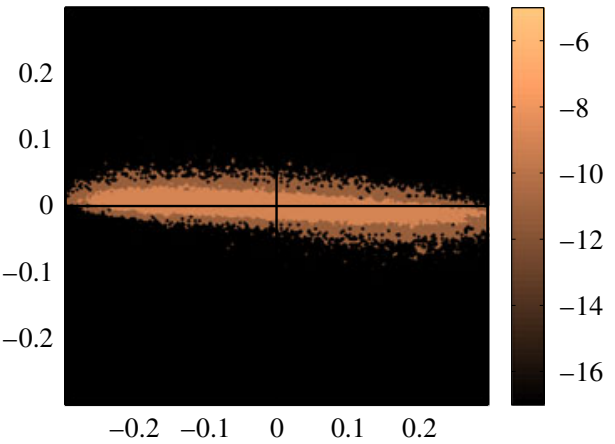

(d)

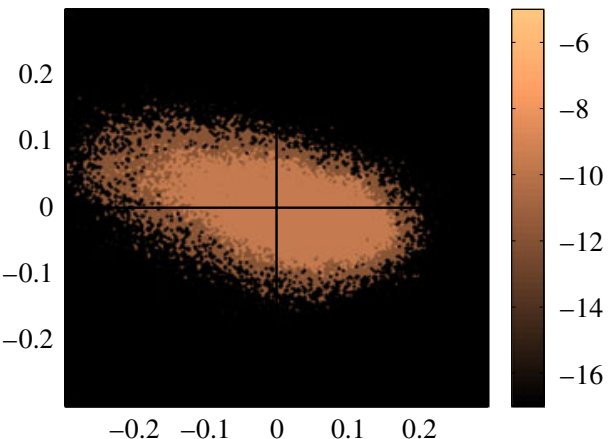

$(f)$

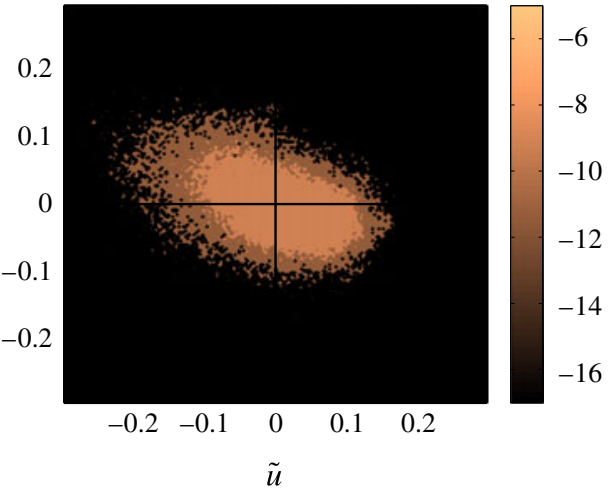

FIGURE 5. (Colour online) Logarithm of the PDF of the streamwise and wall-normal velocity for the outer optimal structures at $t=T_{\text {out }}(a, c, e)$ and for the fully turbulent flow $(b, d, f)$ at different constant $y^{+}$-planes: $y^{+}=10, y^{+}=50, y^{+}=100,(a-f)$.

turbulent flow, shown in the right column of figure 5 for corresponding wall-normal positions. The two set of PDF distributions are very similar, although the data in the left column are obtained by using only the perturbation at a given time, whereas the results in the right column are computed from the statistics of the DNS. On the other hand, the PDF of the inner optimal perturbation (not shown) is quite similar to that extracted from the DNS only at $y^{+}=10, \tilde{u}$ and $\tilde{v}$ rapidly fading away towards the centre of the channel. This indicates that, while the inner optimal disturbance is representative of the self-sustained wall cycle, the outer nonlinear optimal disturbance is representative of bursting events populating the fully turbulent channel flow. 


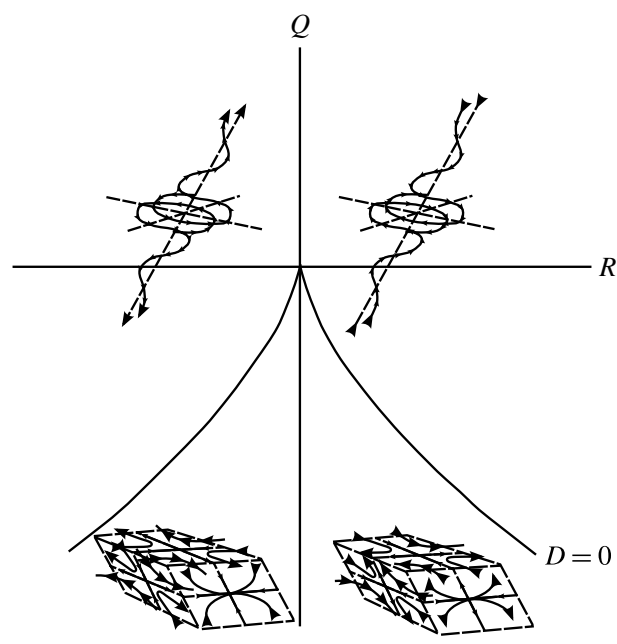

FIGURE 6. Sketch of the flow topologies associated with different regions of the $Q-R$ plane, $Q$ and $R$ being the second and third invariant of the velocity gradient tensor, and $D=(27 / 4) R^{2}+Q^{3}$ (Blackburn, Mansour \& Cantwell 1996).

\subsubsection{Topology analysis}

The outer optimal disturbance has been found to show the same typical features of a bursting event. However, one may wonder whether its complex structure is well representative of the vortical disturbances observed in fully turbulent conditions. In order to answer to this question, we have compared the main vorticity features of the optimal structures with those of the fully turbulent flow (Blackburn et al. 1996). Chong, Perry \& Cantwell (1990) proposed a topological analysis of vortical flow structures based on the first three invariants of the velocity gradient tensor, here referred to as $P, Q$ and $R$, respectively. Incompressible flows being restrained to the $P=0$ space, the flow topologies that can be found in the channel flow considered here can be classified depending on the values of $Q$ and $R$. With reference to figure 6, flow structures which fall in the upper region of the $Q-R$ plane are called stable (left) or unstable (right) focus topologies, representing vortex stretching or compression, respectively; whereas, in the lower region of the plane, stable (left) and unstable (right) node/saddle/saddle topologies are found. The shapes of the local flow field corresponding to these topologies are sketched in figure 6. As described by Blackburn et al. (1996), for a turbulent channel flow, moving from the wall to the centreline of the channel, the PDF of $Q$ and $R$ will vary, indicating the different structures found at different wall-normal positions in the flow. Figure 7 provides the PDF of $Q$ and $R$ for the outer optimal disturbance $(a, c, e)$ and for the DNS of the corresponding turbulent flow $(b, d, f)$ at $y^{+}=10,50,100$. One can notice that in both cases, close to the wall, the PDFs are rather uniformly distributed among all quadrants of the $Q-R$ plane. On the other hand, towards the centreline of the channel, the dominant structures are tube-like, shaped as those sketched in figure 6 above the line $D=0$. Moreover, the PDFs spread mostly in the second and fourth quadrants, indicating a predominance of stable focus/stretching and unstable node/saddle/saddle topologies. As discussed by Blackburn et al. (1996) for the case of turbulent channel flow, stable focus/stretching topologies appear to provide a link between the inner and outer regions of the flow, with structures originating in the viscous sublayer 

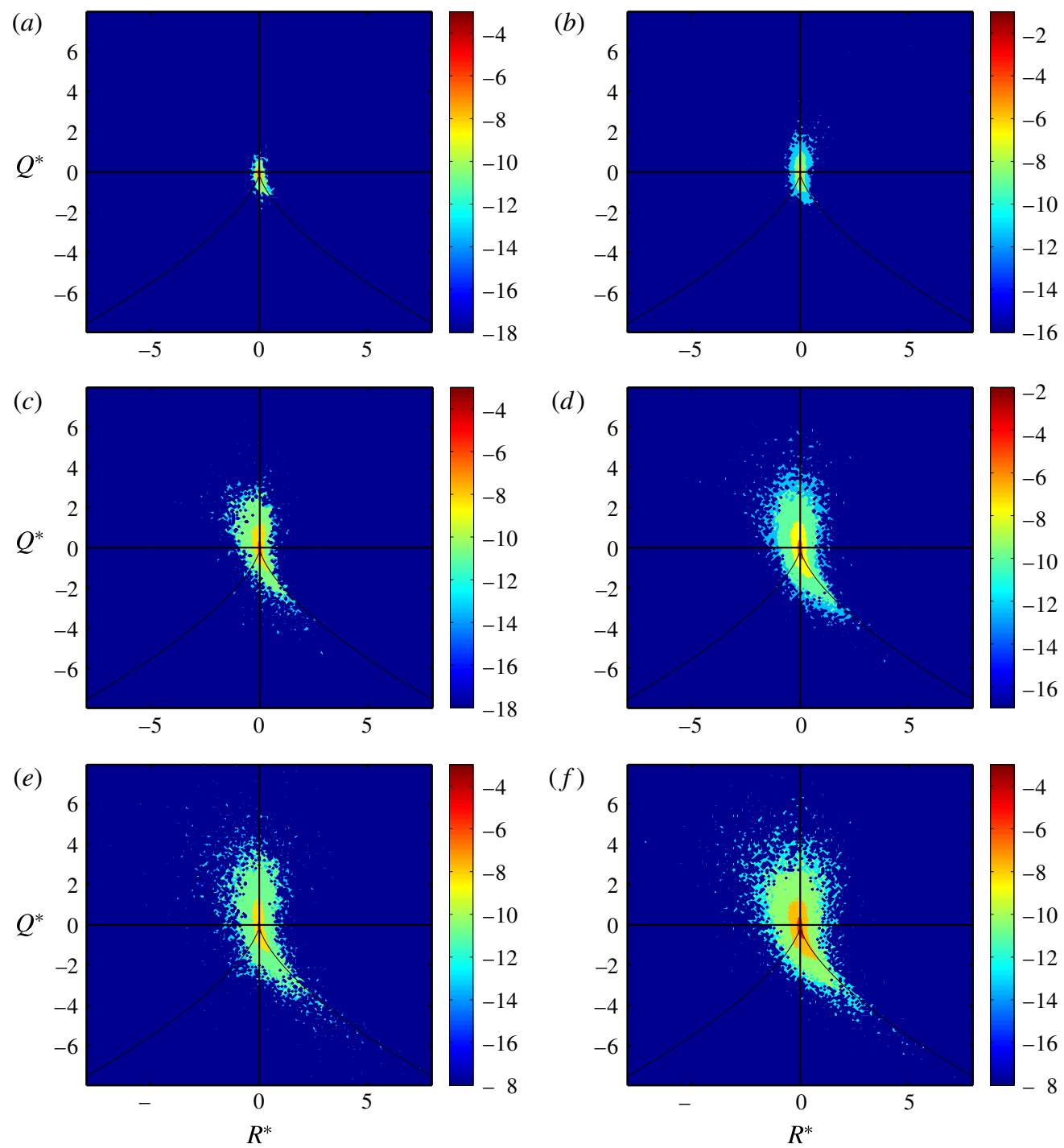

FIgURE 7. (Colour online) Logarithm of the PDF of the non-dimensional second $\left(Q^{*}\right)$ and third $\left(R^{*}\right)$ invariant of the velocity gradient tensor, for the outer optimal structures at $t=$ $T_{\text {out }}(a, c, e)$ and for the fully turbulent flow $(b, d, f)$ at different constant $y^{+}$-plane $\left(a-f: y^{+}=\right.$ $10,50,100)$. Notice that $Q^{*}=Q / Q_{w}$ and $R^{*}=R / Q_{w}^{3 / 2}, Q_{w}$ being the second invariant of the antisymmetric part of the velocity gradient tensor averaged on each $y^{+}$-constant plane.

and extending towards the outer region, mostly associated with hairpin or horseshoe shapes. The presence of hairpin vortices in the outer optimal disturbance and the fact that its $Q-R$ topology distribution reproduces well that of the corresponding fully turbulent flow may indicate the presence of those structures in fully turbulent conditions, at least for the moderate value of the Reynolds number used here. On the other hand, the inner optimal disturbance, which does not show hairpin vortices, is characterized by a different topology distribution, without any clear preference for the stable focus/stretching and the unstable node/saddle/saddle topologies, with the 


$\begin{array}{lcccc} & \lambda_{z}^{+} & y^{+} & \lambda_{x}^{+} & y^{+} \\ t^{+}=T_{i n}^{+} & & & & \\ \hat{E}_{u u} & 113.8 & 13.6 & 189.4 & 9.21 \\ \hat{E}_{v v} & 113.8 & 29.1 & 189.4 & 16.5 \\ \hat{E}_{w w} & 113.8 & 10.2 & 189.4 & 13.6 \\ t^{+}=T_{o u t}^{+} & & & & \\ \hat{E}_{u u} & 189.6 & 14.9 & 757.5 & 74.2 \\ \hat{E}_{v v} & 142.2 & 67.6 & 757.5 & 81.5 \\ \hat{E}_{w w} & 227.5 & 81.5 & 757.5 & 74.2\end{array}$

TABLE 1. Wavelength and corresponding wall-normal position of the peaks of the premultiplied energy density spectrum shown in figure 8.

vortices rapidly fading away far from the wall (not shown). Therefore, the similarity of the outer optimal flow topology with that of the fully turbulent flow suggests that these structures are well representative of the vortical dynamics of a turbulent channel flow from the wall towards the centreline of the channel.

\subsubsection{Spectrum analysis}

We have found that the outer optimal disturbance reproduces well the vortical topology of the corresponding turbulent channel flow; now, we want to investigate whether the size of these vortices and their main wavelengths might be representative of the broadband spectrum of wavelengths typical of turbulent flows. At this purpose, we have extracted the streamwise and spanwise premultiplied energy density spectra from the nonlinear optimal disturbances at $T_{\text {in }}^{+}$and $T_{\text {out }}^{+}$, and compared them with the same spectra extracted from the DNS of the corresponding fully developed turbulent flow. Figure 8 provides the premultiplied spectrum distributions along the wall-normal direction obtained from the DNS (shaded contours) and from the optimal disturbances at $T_{\text {in }}^{+}$(light blue isolines) and at $T_{\text {out }}^{+}$(black isolines). The spanwise $(a, c, e)$ and streamwise $(b, d, f)$ spectra have been obtained for energy densities computed on the basis of the streamwise $(a, b)$, wall-normal $(c, d)$ and spanwise $(e, f)$ components of velocity, $\hat{E}_{u u}, \hat{E}_{v v}$ and $\hat{E}_{w w}$, respectively. The $\mathrm{X}$ and the $\mathrm{O}$ symbols mark the peak values of the energy density spectrum for the inner and the outer optimal structures, respectively. Such peak values are also provided in table 1. For the inner optimal structure, the energy peak is found for $\lambda_{z}^{+}=113.8$, rather close to the wall $\left(y^{+}\right.$ ranging from 10 to 29 depending on the considered energy density), providing the typical spanwise spacing of streaks (Kline et al. 1967). Concerning the streamwise wavelength, the energy peak is at $\lambda_{x}^{+}=189.4$, corresponding to the wavelength of the strong wiggling of the streaks due to the presence of streamwise vortices flanking them. However, looking at all of the streamwise spectra (right column), one can notice a secondary peak at $k_{x}^{+} \approx 0.0058$, corresponding to a secondary wavelength, $\lambda_{x}^{+} \approx 1082.7$, close to the typical streamwise wavelength of streaks in fully turbulent conditions (Kline et al. 1967), and roughly corresponding to the wavelength of the spot-like localization of the optimal structures characterizing the inner optimal perturbation. Nevertheless, for this inner optimal structure, both the streamwise and spanwise spectra appear very narrow, including only a small portion of the broadband range of wavenumbers found by the DNS (compare the light blue isolines with the shaded isocontours). On the other hand, the spectra computed for the outer optimal 

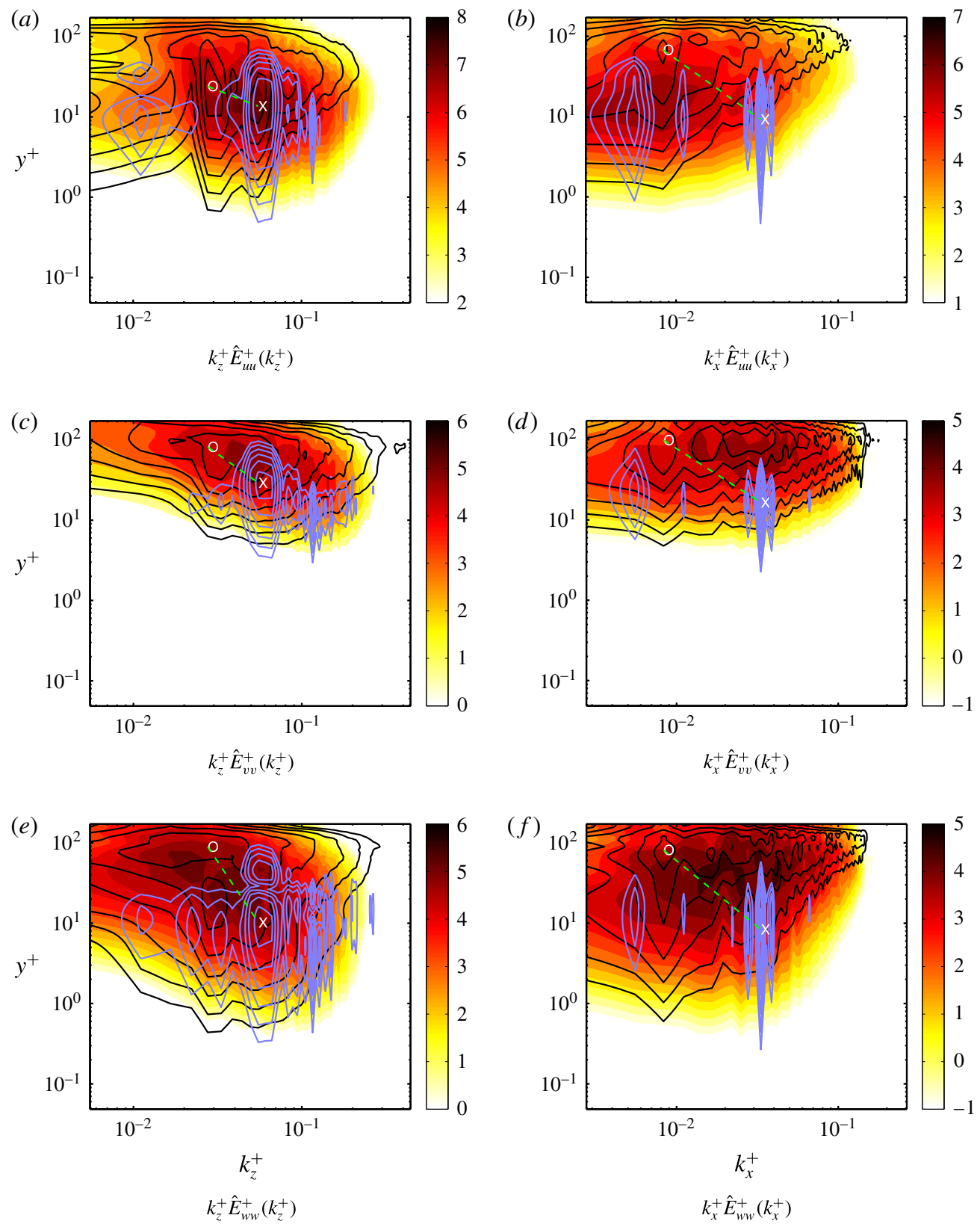

FIGURE 8. (Colour online) Logarithm of the premultiplied power spectrum versus the wall-normal distance $y^{+}$for the DNS (shaded contours), inner optimal solution (blue isolines) and outer optimal solution (black isolines) at target time. The symbols $\mathrm{X}$ and $\mathrm{O}$ indicate the maximum value for the inner and outer peak, respectively. The green dotted line joining the inner and outer energy peak provides the scaling laws $y^{+}=c\left(k_{x, z}^{+}\right)^{-1}$, with slopes (a) $c=0.0921,(b) c=0.4608,(c) c=0.6970,(d) c=0.1028,(e) c=0.1439$, (f) $c=0.1287$.

have energy peaks at larger wavelengths in $x$ and $z$, with $\lambda_{x}^{+}=757.5$ well reproducing the peak value found by DNS in the $\hat{E}_{u u}$ spectrum, probably corresponding to the 
streak streamwise spacing found in the outer optimal disturbance. Moreover, the outer optimal perturbation spectra are almost overlapped with those extracted from the DNS (compare the black isolines with the shaded contours), indicating that the optimal structure computed for $T_{\text {out }}^{+}$is well representative of the turbulent motion in the same flow condition.

Concerning the typical wavelength of the coherent structures in fully turbulent flow, the attached eddy theory of Townsend (1980) suggests that the size of the typical eddies in a turbulent shear flow scales with the wall-normal direction, the smallest-eddy dimension scaling with inner units $\left(l^{+}\right)$, and the largest-eddy dimension scaling with outer units, in both streamwise and spanwise directions. In order to verify whether this hypothesis is valid also for the optimal structures found here, we have considered the energy peaks of the premultiplied energy spectra, marked in figure 8 by $\mathrm{X}$ and $\mathrm{O}$ for the inner and outer optimal disturbance, respectively. Tracing a straight line between these two peaks (green dashed line), we can infer a scaling of the form $y^{+} \propto \lambda_{x, z}^{+}\left(y^{+} \propto\left(k^{+}{ }_{x, z}\right)^{-1}\right)$, as conjectured by Townsend (1980). Concerning the scaling coefficients, provided in the caption of figure 8 , we have obtained values very close to those available in the literature for all components of the energy (Hoyas \& Jiménez 2006; Hwang 2015). Thus, the scaling laws extracted on the basis of the inner and outer energy peaks reproduce well the scalings found in fully turbulent flows. However, we must remark that at the considered moderate Reynolds number a distinct scale separation in the streamwise direction is not established yet, data at higher Reynolds numbers being needed for confirming the results (del Álamo \& Jiménez 2003).

\subsection{Time evolution of the outer optimal perturbation}

\subsubsection{Production and dissipation analysis}

The optimal structure arising at the outer time scale is structurally rather complex and the mechanisms leading to a strong energy growth cannot be simply related to a large-scale cycle similar to that characterizing the inner scale (Hwang \& Cossu 2010b). Insight into the energy growth mechanisms can be gained by considering the time evolution of the energy density of the structures. Scalar multiplication of (2.1) by $\tilde{\boldsymbol{u}}$ provides the following Reynolds-Orr equation (Schmid \& Henningson 2012) for the disturbance:

$$
\frac{1}{2} \frac{\partial E}{\partial t}=\int_{V} \underbrace{-\tilde{\boldsymbol{u}} \cdot(\tilde{\boldsymbol{u}} \cdot \nabla \boldsymbol{U})}_{P} \mathrm{~d} V-\int_{V} \underbrace{\frac{1}{\operatorname{Re} \nabla \tilde{\boldsymbol{u}}: \nabla \tilde{\boldsymbol{u}}}}_{D} \mathrm{~d} V+\int_{V} \underbrace{\tilde{\boldsymbol{u}} \cdot \nabla \cdot\left(\frac{\overbrace{\tilde{\tilde{u}} \tilde{\boldsymbol{u}}}^{\tau}}{\tau}\right.}_{P_{\tau}} \mathrm{d} V,
$$

where $P(x, y, z, t)$ is the energy production, $D(x, y, z, t)$ is the energy dissipation and $P_{\tau}(x, y, z, t)$ is the contribution of the Reynolds stress to the production. Figure $9(a)$ shows the time evolution of these three terms integrated over the whole computational domain, denoted hereafter as $P_{V}, D_{V}$ and $P_{\tau V}$. First of all, the strong energy peak, followed by an increase of the dissipation $D_{V}$, confirms that the transient evolution of this optimal structure can be interpreted as a strong energy burst, which is then dissipated in time reaching a dissipation peak at $t^{+} \approx 3 T_{\text {out }}^{+}$. Such a time interval is in agreement with the large-scale temporal oscillation observed by Hwang \& Bengana (2016) for the largest attached eddy and recognized as a bursting event by Flores \& Jimenez (2010). One can notice the non-negligible contribution of the term $P_{\tau V}$ in the early time evolution of the perturbation, except at very small times, when 

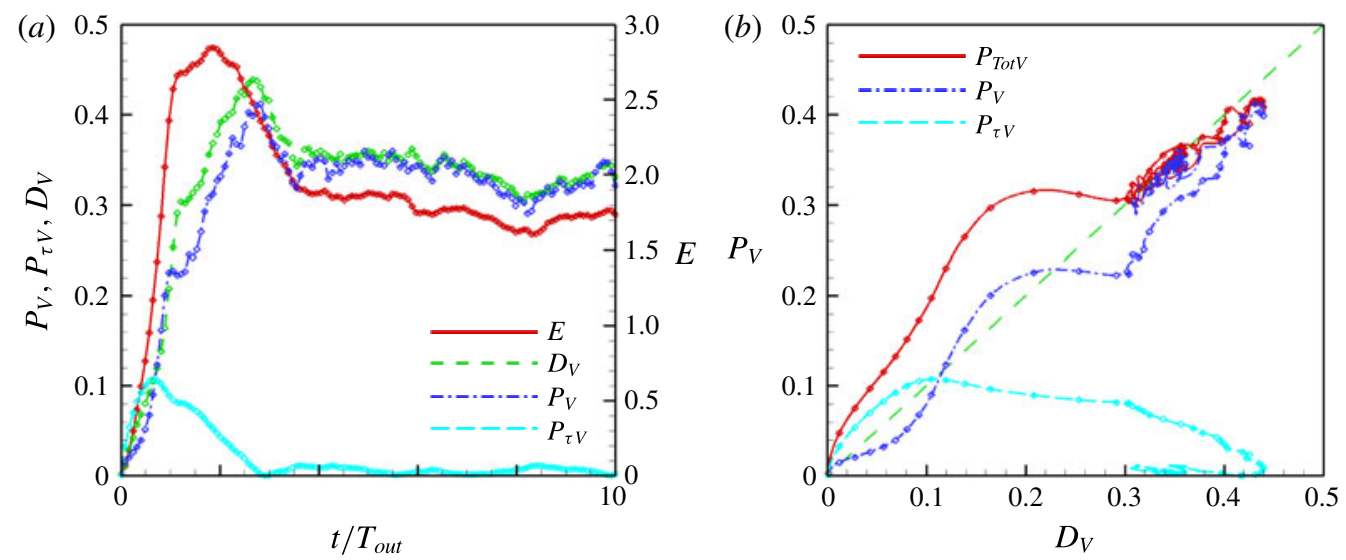

Figure 9. (Colour online) (a) Time evolution of the energy $E$ (red), production $P_{V}$ (blue), Reynolds stress production $P_{\tau V}$ (cyan), and dissipation $D_{V}$ (green). (b) Trajectories in the plane $D_{V}-P_{V}$ of the production $P_{V}$, Reynolds stress production $P_{\tau V}$, and total production $P_{T o t V}=P_{V}+P_{\tau V}$. The time interval between symbols is equal to $\Delta t^{+}=24.5$.

the main production mechanism is the Orr mechanism (Orr 1907), as inferred by analysing the time evolution of the optimal structures provided in $\S 3.2 .2$. This points out the important role of the Reynolds stress in the dynamics of the perturbation. It is noteworthy that this result is in agreement with the analysis of Jiménez (2015) who estimates that the effect of the linear energy growth due to the Orr mechanism is dominant for $\bar{t}=t^{+} / R e_{\tau}<0.15$. In our case, $\bar{t}_{\text {Orr }} \approx 0.136$ and $\bar{T}_{\text {out }}=T_{\text {out }}^{+} / R e_{\tau}=1.694$, therefore, the linear limit is less than one-tenth of the target time. Figure $9(a)$ shows that in the first part of the bursting event a strong linear growth can be observed followed by a larger nonlinear growth till $t^{+}=2 T_{\text {out }}^{+}$. In particular, the contribution of the Reynolds stresses increases till $t^{+} \approx T_{\text {out }}^{+}$and becomes negligible for $t^{+}>2 T_{\text {out }}^{+}$. In fact, the term $P_{\tau V}$ reaches its maximum approximately at the target time, when the generation of the largest hairpin vortices is completed, and then it decays in time, leading to the establishment of featureless turbulence. This behaviour can be better observed in figure $9(b)$ providing the projection of the time evolution of the perturbation onto a production-dissipation plane. Starting close to the origin, the trajectory reaches the peak of the total production $P_{V}+P_{\tau V}$ due to the successive increase of $P_{\tau V}$ and $P_{V}$, before starting to oscillate around the point $P_{V} \approx D_{V} \approx 0.35$, representing the turbulent self-sustained state.

In order to analyse the energy production and dissipation mechanisms, we evaluate the energy exchange in the wall-normal direction by expressing the Reynolds-Orr equation using the Cartesian notation (Jiménez 1999):

$$
\left(\partial_{t}+U_{j} \partial_{j}-\frac{1}{\operatorname{Re}} \nabla^{2}\right) \frac{\tilde{u}_{i} \tilde{u}_{i}}{2}+\partial_{j}\left(\tilde{u}_{j}\left(\tilde{p}+\frac{\tilde{u}_{i} \tilde{u}_{i}}{2}\right)\right)=-\tilde{u}_{i} \tilde{u}_{j} \partial_{j} U_{i}-\frac{1}{\operatorname{Re}}\left(\partial_{j} \tilde{u}_{i}\right)^{2}+\tilde{u}_{i} \partial_{j} \tau_{i j} .
$$

Integrating this equation in the streamwise and spanwise direction, we obtain an equation for the wall-normal transport of energy, allowing one to identify the last term on the left-hand side as the wall-normal energy flux, $\Phi=\tilde{v}\left[\tilde{p}+\left(\tilde{u}^{2}+\tilde{v}^{2}+\tilde{w}^{2}\right) / 2\right]$, and the first two terms on the right-hand side as the production and dissipation terms (whose integral counterparts are denoted as $P_{x, z}$ and $D_{x, z}$, respectively). The net 

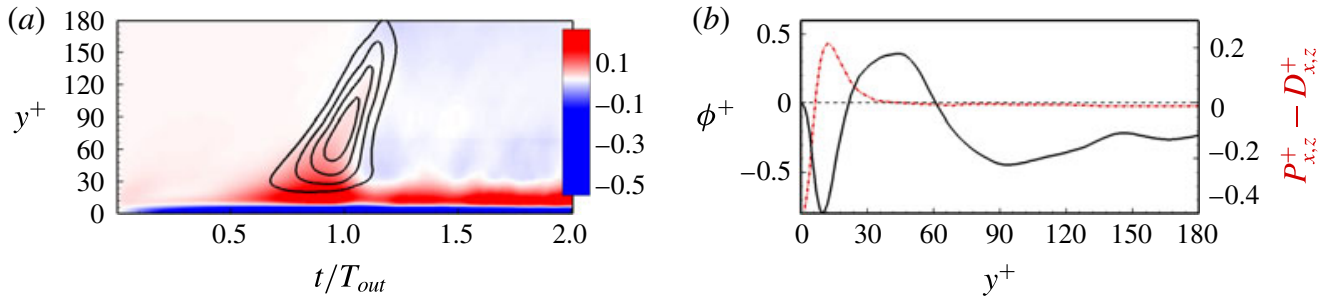

FIgURE 10. (Colour online) (a) Time evolution of the net local energy production, given by the difference between the production and dissipation at each $y^{+}$(shaded contours), and of the wall-normal energy flux (isolines for $\Phi^{+}=1,2,3,4$, from the outermost to the innermost contour) for the outer optimal structure. (b) Net local energy production (red dashed line) and wall-normal flux (black solid line) versus $y^{+}$, extracted at $t^{+}=2 T_{\text {out }}^{+}$, showing a production peak in the inner zone $\left(y^{+} \approx 20\right)$, whereas the outer region is characterized by a weak dissipation.

energy production at a given $y^{+}$-constant plane, provided by the difference $P_{x, z}-D_{x, z}$, is plotted versus time in figure 10(a) (shaded contours). As found for fully developed turbulent flows at comparable values of $\operatorname{Re}_{\tau}$ (Jiménez 1999), the net production of turbulent energy (red contours) is well localized at the inner scale $\left(y^{+} \approx 20\right)$ and extends to the outer layer for times smaller than the target time indicating that both inner and outer structures contribute to the perturbation energy increase. The isolines for positive wall-normal energy flux $\left(\Phi^{+}=1,2,3,4\right)$ are provided in figure $10(a)$, clearly indicating an outward flux of energy towards the centre of the channel for $t^{+} \approx T_{\text {out }}^{+}$, corresponding to the formation of the hairpin vortex. Whereas, at the centre of the channel, dissipation is found to exceed production, and the flux continuously decreases becoming slightly negative, as one can observe in figure $10(b)$, showing the excess of local energy production and the wall-normal flux extracted at $t^{+}=2 T_{\text {out }}^{+}$. This indicates the presence of a coherent inverse-cascade process (Jiménez 1999) typical of moderate values of $R e_{\tau}$, in which energy is transferred from the inner scales at the wall, i.e. the streaks, to large-scale dissipating structures in the outer layer, the hairpin vortices. Notice that at the considered value of $R e_{\tau}$, when fully turbulent flow is achieved, no energy production is observed far from the wall, whereas at larger values of $\operatorname{Re}_{\tau}$ a (weaker) production peak is observed also at the outer scale (see Lee \& Moser 2015). The large-scale dissipating hairpin structures will eventually breakdown, transferring the energy to incoherent small-scale fluctuations, closing the loop. This wall-normal energy transfer occurring in a short time is thus linked to the occurrence of a transient energy peak which appears in the form of a rapid ejection (positive wall-normal velocity $\tilde{v}$ ) followed by a longer sweep (negative $\tilde{v}$ ), similar to a typical bursting event.

\subsubsection{Analysis of the flow structures}

To further characterize the dynamics of the outer nonlinear optimal perturbation, we analyse its time evolution. Figure 11 provides 10 snapshots of the perturbation ( $Q$-criterion isosurfaces, coloured by the wall-normal distance), from $t^{+}=0$ to $t^{+}=431$. The initial perturbation is localized in the three space directions and is composed of two packets of thin counter-rotating vortices showing a spanwise symmetry, placed at $y^{+} \approx 20$ (as indicated by the colours in figure $11 a$ ). Even if the optimization is based on the outer time scale, the core of the vortical structures 
(a)
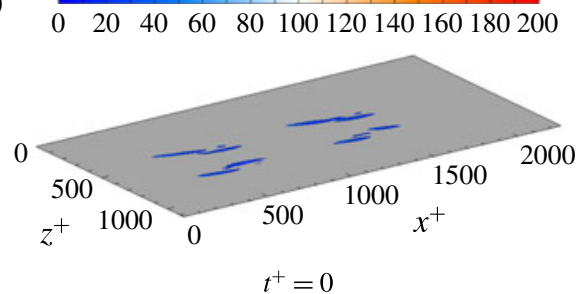

(c)

$$
\begin{array}{llllll}
0 & 20 & 40 & 60 & 80 & 100120140160180200
\end{array}
$$

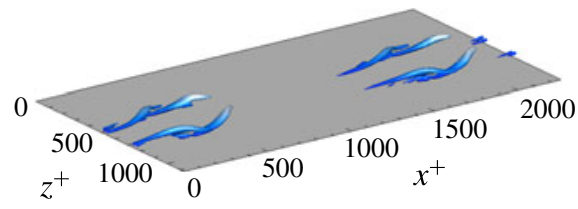

$$
t^{+}=98
$$

(e)
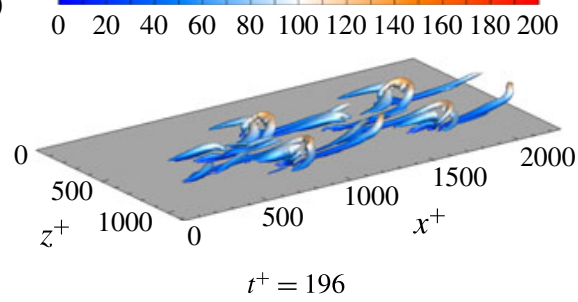

(g)
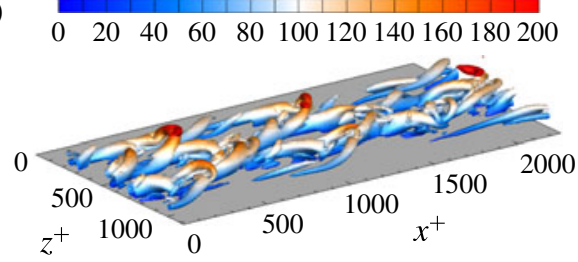

$$
t^{+}=294
$$

(i)

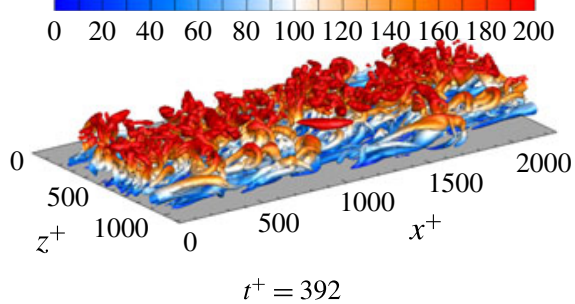

(b)
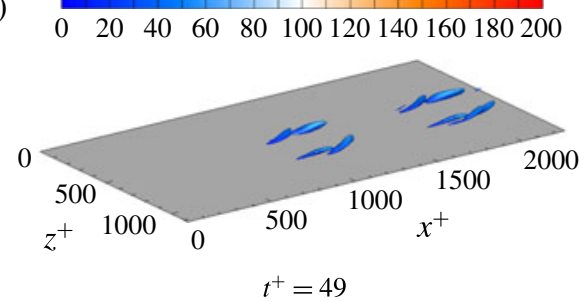

(d)
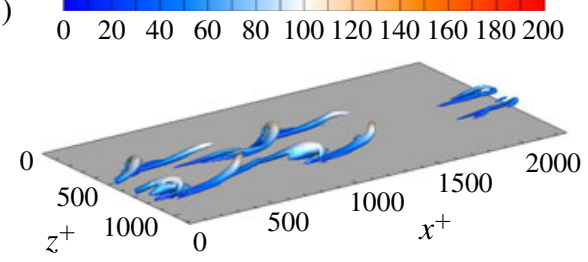

$$
t^{+}=147
$$

(f)
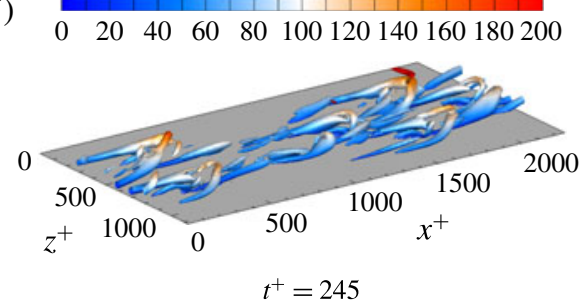

(h)
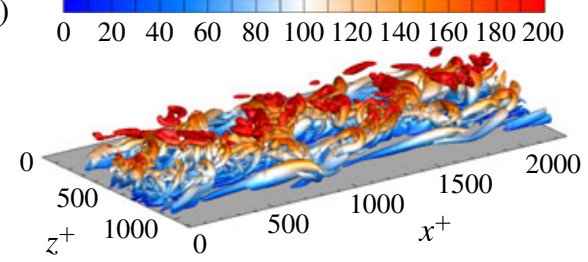

$$
t^{+}=343
$$

(j)

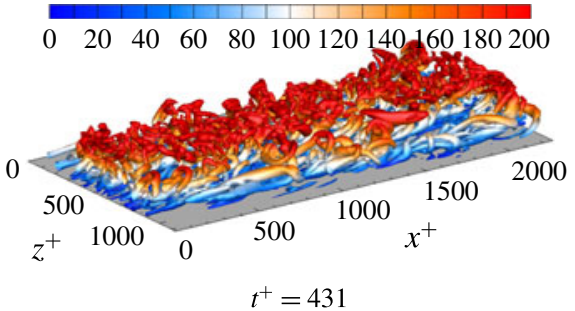

FIGURE 11. (Colour online) Snapshots of the time evolution of the outer optimal structures: isosurfaces of $Q$-criterion coloured by the wall-normal distance $y^{+}$.

at initial time appears to be in the inner region, the main part of the energy of the spanwise and wall-normal velocity being located at $y^{+} \approx 10-40$ (whereas at $t^{+}=T_{\text {out }}^{+}$the energy peak is at $y^{+}>36$ or $y>0.2$, as provided in table 1 ). Whereas, the streamwise velocity perturbation is located far away from the wall at $y^{+} \approx 60$ (at $t^{+}=0$ ). In figure $11(b)$ one can observe the typical downstream tilting due to the Orr mechanism (Orr 1907). This initial phase of the energy growth 

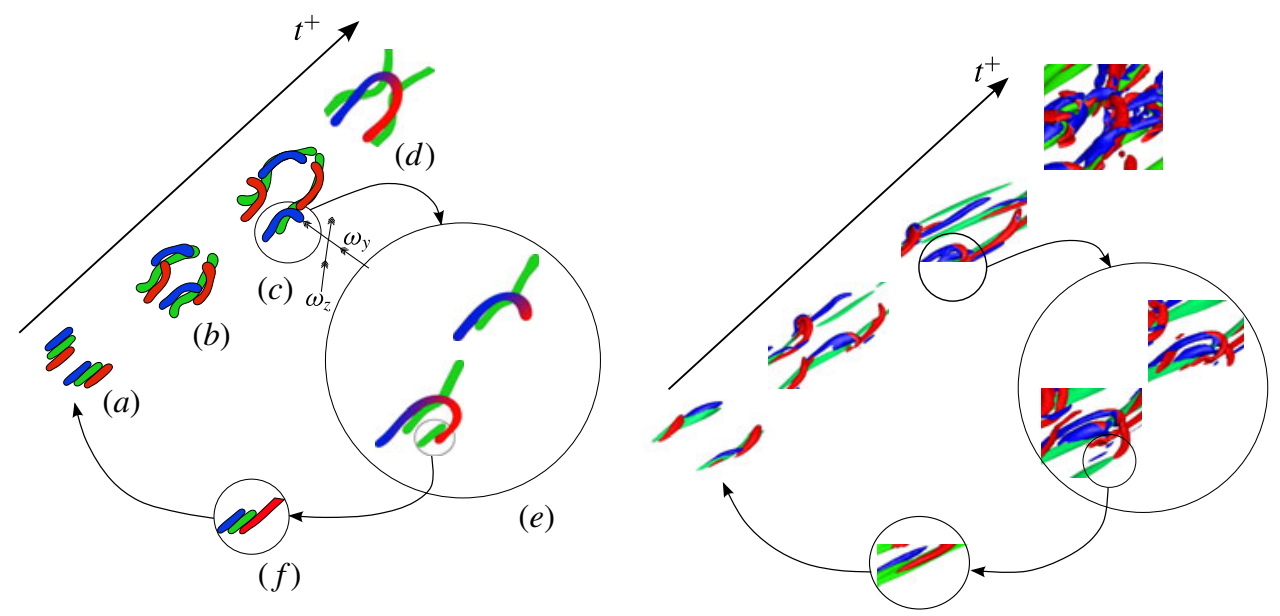

FIGURE 12. (Colour online) (Left) Sketch of the main steps of the dynamics of the outer optimal perturbation based on the snapshots (right) extracted at $(a) t^{+}=49,(b) t^{+}=123$, (c) $t^{+}=147.5,(d) t^{+}=294,(e) t^{+}=172,196.5,(f) t^{+}=221$. Isosurfaces of negative streamwise velocity (green) and $Q$-criterion coloured by the values of streamwise vorticity (blue for positive, red for negative).

agrees with the linear analysis of Jiménez (2015) who demonstrates that for very short times $\left(\bar{t}=t^{+} / R e_{\tau}<0.15\right)$ the energy growth due to the Orr mechanism is dominant. Following the evolution of the perturbation, we can notice that the vortices tend to be lifted up from the wall towards the centre of the channel, developing structures of increasing size in an inverse cascade from small to large scales (Jiménez 1999). Concerning the vortical dynamics, one can observe the formation of new vortices aligned with the initial ones along modulated streamwise streaks (see figure $11 c, d$ ). These vortices are lifted in the wall-normal direction, creating symmetric or non-symmetric arches on top of the negative streaks at the wall (Wang, Huang \& Xu 2015), as one can observe in figure $11(d, e)$. Once the small-scale hairpin and cane vortices have been created, some of them further grow and lift in the outer region, merging with the nearest vortices in large-scale symmetric hairpin vortices whose head is placed between two streamwise streaks at the wall (Adrian, Balachandar \& Lin 2001), as shown in figure $11(f, g)$, generating a new weaker large-scale negative streak between the hairpin legs. Once the structures have reached their maximum spatial growth, corresponding approximately to the energy peak in figure $9(a)$, the structures begin to break down, starting an energy cascade from the large scales towards the small ones, closing the loop (figure $11 h, i, j$ ).

Figure 12 summarizes the main steps of the time evolution of the outer optimal perturbation: we conjecture it is representative of a bursting event which transfers the energy from small- to large-scale structures, and then back towards smallscale perturbations. The left panel provides a sketch of the main steps of the evolution shown by seven successive snapshots on the right panel, for $t^{+}=$ $49,123,147.5,172,196.5,221,294$, respectively. An initial perturbation leading to a bursting event is originated by two flanking pairs of small counter-rotating vortices (red and blue isosurfaces at the bottom left angle of both panels), which are the first elements of the wall self-sustained cycle (Waleffe 1997). These vortices initially increase their energy by the Orr mechanism and then generate low-speed 
streaks by the lift-up mechanism (Landahl 1980) (green isosurfaces, step $(b)$ ). These streaks increase their amplitude, and exhibit secondary sinuous or varicose instability (Andersson et al. 2001). As a result, the initial streamwise vortices bend over the streak at the point where the instability is triggered, forming arch-shaped structures (Schoppa \& Hussain 2002; Wang et al. 2015) (step c). Being very close to each other and continuously lifting in the wall-normal direction, two of these non-symmetric arch-shaped vortical structures merge together, generating a large symmetric hairpin structure (blue and red isosurfaces, step $d$ ). This large-scale hairpin vortex increases in size up to the centreline of the channel and then begins to break down dissipating the energy; instead, the non-symmetric small-scale vortices on top of the streaks may induce inflectional instabilities on the instantaneous velocity profile. In particular, strong low-speed streaks may induce an inflection point in the $x-y$ plane, triggering a Kelvin-Helmholtz-type instability producing spanwise vorticity, such as the arch of the hairpin vortices, but also inflection points in the $x-z$ plane, producing wall-normal vorticity (Heist, Hanratty \& $\mathrm{Na} 2000$ ). The tilting of the wall-normal vorticity by the mean velocity profile induces streamwise vortices of opposite sign with respect to the initial one (step e), as discussed by Heist et al. (2000). This new counter-rotating vortex flanks the initial one, being able to create a new low-speed streak by the lift-up mechanism (step $f$ ), and the loop can restart again from the small-scale structures towards the large-scale ones. Concerning the time scales typical of this loop, the complete formation of the small arch vortices participating to the self-sustained cycle takes approximately $\Delta t^{+} \approx 150$ time units (see figure $11 c-i$ ) as in Wang et al. (2015), whereas the complete formation of the train of large-scale hairpin vortices takes approximately $\Delta t^{+} \approx 300$ time units, which corresponds approximately to the target time $T^{+}$, similarly to the observations of Zhou et al. (1999) where the hairpin packets have spacing approximately equal to $\lambda_{x}^{+} \approx 450$ (see figure 3 ). Notice that the self-sustained cycle mainly involves streaks and vortices close to the wall, but it transiently induces large-scale hairpin vortices as a by-product of its evolution. These large vortical structures have the role of realizing an inverse energy cascade reaching the outer scale and dissipating the stored energy towards smaller scales, allowing a new wall cycle to be established. It appears thus that the large-scale hairpin vortices observed in the nonlinear optimal disturbance do not directly participate into the self-sustained cycle, but they are transient dynamical features which ensure the occurrence of energy peaks and subsequent dissipation typical of bursting events.

\section{Conclusion}

It is known that, behind its chaotic dynamics, turbulent flow is populated by coherent structures, i.e. flow motions highly correlated over both space and time, carrying a large part of the flow momentum. In the present study, for the first time, a recently developed nonlinear optimization technique based on Lagrange multipliers is employed to unravel the dynamics of such structures.

In particular, a nonlinear transient growth analysis has been performed to study which kind of coherent structures are able to trigger rapid events with a strong energy growth, similar to bursting events, in a canonical wall-bounded turbulent flow such as the channel flow. The optimization procedure, focusing on the dynamics of finiteamplitude disturbances, provides different nonlinear optimal structures depending on the chosen time scale for the energy growth.

For an inner time scale, corresponding approximately to one eddy turnover time evaluated in the buffer layer at $y^{+}=19$ (the superscript ${ }^{+}$indicating variables 
expressed in inner units), nonlinear optimal structures consist of highly modulated streaks having a typical spanwise spacing $\lambda_{z}^{+} \approx 113$, surrounded by positive and negative streamwise vortices, with a spanwise spacing $\lambda_{z}^{+} \approx 56$. These nonlinear optimal structures, localized in space in a spot-like fashion, well represent the self-sustained cycle of turbulence at the wall: linearly growing streaks saturate and oscillate due to secondary instability, regenerating new localized quasi-streamwise vortices by nonlinear coupling.

For the outer time scale, corresponding approximately to one eddy turnover time at the centreline of the channel, a much more complex optimal structure is observed, mostly composed of packets of hairpin vortices on top of highly oscillating streamwise streaks. In particular, strong vortical and streaky structures are observed at different spatial scales, ranging from the wall to the outer layer. The probability density function of the velocity disturbance characterizing such an outer optimal is concentrated in the second and fourth quadrants of the streamwise versus wall-normal velocity plane. This indicates the prevalence of ejection and sweep events, as happens in a strong bursting event. Moreover, we show that the outer most energetic structure well describes the wavenumber spectrum, the vortical topology, and the production-dissipation wall-normal distribution typical of turbulent flows at moderate Reynolds numbers. Whereas, the inner optimal structure includes only a small portion of the broadband range of wavenumbers and vortical topologies found at different wall-normal positions by a direct numerical simulations of the fully turbulent flow. The analysis of the distribution of the most energetic wavelengths in the wall-normal direction for the inner and outer optimal disturbances shows that the optimal structures computed here scale in size accordingly to the attached eddy theory of Townsend (1980). In particular, the spatial scaling laws extracted on the basis of the inner and outer energy peaks reproduce well the scalings found in fully turbulent flows.

Finally, a careful analysis of the time evolution of the optimal flow structures has been performed, providing the dynamics of the initial perturbations leading to a bursting event. An optimal bursting event is originated by two flanking pairs of small counter-rotating vortices at the wall, the basic elements of the wall self-sustained cycle; due to the Orr and lift-up mechanisms, these vortices are able to generate strong low-speed streaks, which exhibit secondary sinuous or varicose instability; as a result, the initial streamwise vortices bend over the streak at the point where the instability is triggered, forming arch-shaped structures which lift in the wall-normal direction and merge together, generating large symmetric hairpin structures; the large-scale hairpin vortices increase in size up to the centreline of the channel and then breakdown dissipating the energy; instead, the small-scale vortices on top of the wall streaks are bent and tilted by the mean flow, leading back to the creation of a pair of streamwise vortices, restarting the cycle. Thus, the self-sustained cycle at the wall appears to be the main source of energy of the bursting event, which transiently induces large-scale hairpin vortices as a by-product; these large vortical structures have the role of realizing an inverse energy cascade reaching the outer scale and eventually dissipating the stored energy towards smaller scales, allowing a new wall cycle to develop. This implies that hairpin vortices, even if inherently transient coherent structures (see Eitel-Amor et al. 2015), are robust features of turbulent channel flows, at least at moderate friction Reynolds numbers, arising as a result of a strong nonlinear instability that repeats in time as a by-product of the self-sustained wall cycle.

These results show that, despite the main source of turbulent energy being located close to the wall and sustained by the wall cycle (Waleffe 1997), for moderate values 
of $R e_{\tau}$ the turbulent motion is characterized by a complex energy transfer which involves inner and outer scales.

In conclusion, this study provides an explanation for the recurrence of energy bursts revealing that they correspond to optimal energy flow structures embedded in the fully turbulent flow. These optimal structures reproduce well the spatial spectra as well as the probability density function of the velocity typically measured in turbulent flows, recovering the mechanism of direct-inverse energy cascade. These results represent an important step towards understanding the dynamics of turbulence and paves the way to new nonlinear techniques to manipulate and control the self-sustained turbulence dynamics. A further challenge will be to extend this analysis to larger values of $R e_{\tau}$, for which a clear scale separation is observed in the spatial spectra and a second peak of energy production exists at the outer scale. This will allow to model the inner-outer interaction under different operating conditions, aiming at understanding the universal mechanisms underlying the turbulent coherent motion. Moreover, further work will aim at exploring the optimal dynamics of coherent structures for other types of turbulent flows such as the boundary-layer flow.

\section{Acknowledgements}

This work was granted access to HPC resources of IDRIS under allocation $x 20162 a 6362$ made by GENCI (Grand Equipement National de Calcul Intensif). The authors would like to thank J.-C. Loiseau for his contribution to the implementation of the optimization algorithm in the code Nek5000 and the anonymous referees for their valuable suggestions. The authors also wish to acknowledge the computational resources of the PrInCE project (grant PONa3-00372-CUP D91D11000100007) at Politecnico di Bari.

\section{Appendix}

In order to derive the equations governing the dynamics of perturbations of the mean turbulent velocity profile in a plane channel flow, we employ a Reynolds decomposition approach similar to that used by Eitel-Amor et al. (2015). The instantaneous flow vector $\boldsymbol{q}=[\boldsymbol{u}, p]^{\mathrm{T}}$, where $\boldsymbol{u}$ is the velocity vector and $p$ is the pressure, is decomposed into a mean flow component $\boldsymbol{Q}=[U, 0,0, P]^{\mathrm{T}}$ and a disturbance $\tilde{\boldsymbol{q}}=[\tilde{u}, \tilde{v}, \tilde{w}, \tilde{p}]^{\mathrm{T}}$ :

$$
\boldsymbol{q}(x, y, z, t)=\boldsymbol{Q}(y)+\tilde{\boldsymbol{q}}(x, y, z, t) .
$$

Injecting this decomposition in the NS equations and averaging over a long time, the following Reynolds-averaged NS equations are obtained:

$$
\boldsymbol{U} \cdot \nabla \boldsymbol{U}=-\nabla P+\frac{1}{R e} \Delta \boldsymbol{U}-\nabla \cdot \overline{\tilde{u} \tilde{u}}
$$

- denoting long-time averaging. Subtracting the time-averaged equations (A 2) from the NS equations provides the following final formulation for the dynamics of the disturbances:

$$
\left.\begin{array}{c}
\frac{\partial \tilde{\boldsymbol{u}}}{\partial t}+\tilde{\boldsymbol{u}} \cdot \nabla \tilde{\boldsymbol{u}}+\boldsymbol{U} \cdot \nabla \tilde{\boldsymbol{u}}+\tilde{\boldsymbol{u}} \cdot \nabla \boldsymbol{U}=-\nabla \tilde{p}+\frac{1}{R e} \Delta \tilde{\boldsymbol{u}}+\nabla \cdot \overline{\tilde{\boldsymbol{u}} \tilde{\boldsymbol{u}}} \\
\nabla \cdot \tilde{\boldsymbol{u}}=0,
\end{array}\right\}
$$



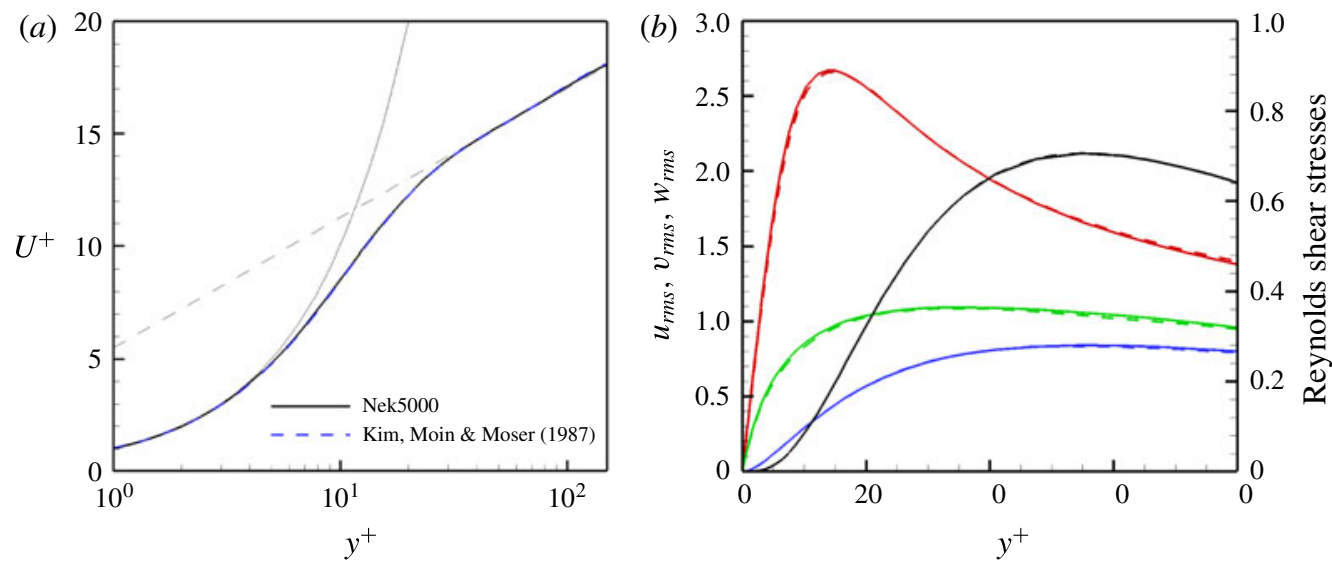

FIgURE 13. (Colour online) (a) Mean velocity profile $U^{+}$versus the wall-normal coordinate $y^{+}$(blue thick lines) obtained by the present DNS (solid) compared with the results of Kim et al. (1987) (dashed). The black thin lines are the linear (solid) and logarithmic (dashed) profiles. (b) Root-mean-square of $\tilde{u}$ (red), $\tilde{v}$ (blue), $\tilde{w}$ (green) and Reynolds shear stress $\tilde{\tilde{u}} \tilde{v}$ (black) normalized by the wall shear velocity, versus $y^{+}$obtained by the present DNS (solid) compared with the results of Kim et al. (1987) (dashed).

where $R e=U_{c} h / v, U_{c}$ indicating the steady centreline velocity magnitude in the plane channel. The last term of the momentum equation is the divergence of the Reynolds stress tensor $\boldsymbol{\tau}=\overline{\tilde{\boldsymbol{u}} \tilde{\boldsymbol{u}}}$ forcing the mean turbulent velocity profile (see (A 2)).

This term appears when the perturbative formulation employs a base flow $\boldsymbol{U}$ which is not a solution of the steady Navier-Stokes equations. Reynolds stresses need to be known or modelled for closing the governing equations: in this work we compute them a priori by a DNS of the fully developed turbulent flow. For this purpose, firstly, the mean flow velocity $\boldsymbol{U}$ is computed by DNS averaging the instantaneous velocity over a long time interval and over the two homogeneous directions, obtaining the velocity profile shown in figure 13(a) (solid thick line), which is compared to the mean flow computed by Kim et al. (1987) (dashed thick line). Then, subtracting the computed mean flow from the instantaneous velocity field, we obtain the perturbation $\tilde{\boldsymbol{u}}$, which contains both coherent and fluctuating parts of the disturbances. The product $\tilde{\boldsymbol{u}} \tilde{\boldsymbol{u}}$ is averaged in time and over the two homogeneous directions. Figure $13(b)$ provides the root-mean-square of $\tilde{u}, \tilde{v}, \tilde{w}$, as well as the Reynolds shear stress $\overline{\tilde{u} \tilde{v}}$ (solid lines) extracted from the DNS, showing an excellent agreement with the same quantities computed by Kim et al. (1987) (dashed line). Based on these data, we achieve a direct evaluation of the Reynolds stress tensor $\boldsymbol{\tau}=\overline{\tilde{\boldsymbol{u}} \tilde{\boldsymbol{u}}}$, whose divergence is shown in figure 14(a) (the three solid lines showing the $x, y$ and $z$ components of $\nabla \cdot \boldsymbol{\tau}$ versus the wall-normal coordinate $y^{+}$). The computed Reynolds stress tensor is employed as a forcing term in (2.1) for the optimization process, providing the inner and outer optimal perturbations. As a consistency check, it is also worth verifying that the computed optimal perturbations satisfy the long-time constraint implicitly imposed by forcing the NS equations with the Reynolds stress tensor. In figure $14(b)$ one can observe the time evolution of the energy of $\nabla \cdot[\tilde{\boldsymbol{u}} \tilde{\boldsymbol{u}}]$, $\tilde{\boldsymbol{u}}$ being the inner (green line) or the outer (red line) optimal perturbation, and [•] denoting the spatial average in the $y$-constant planes. It appears that, after a short 

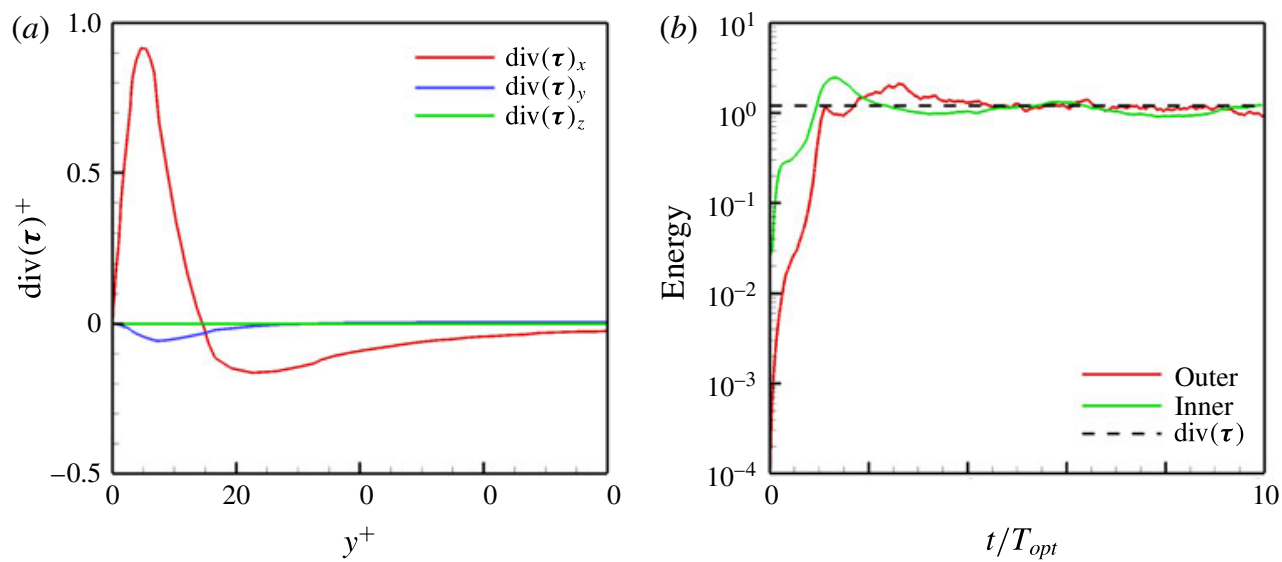

FIGURE 14. (Colour online) (a) Streamwise (red), wall-normal (blue) and spanwise (green) components of the divergence of the Reynolds stress tensor $\tau$ versus $y^{+}$obtained by the present DNS. (b) Energy of $\nabla \cdot[\tilde{\boldsymbol{u}} \tilde{\boldsymbol{u}}]$ (where [•] denotes the spatial average in the $y$-constant planes) for the outer (red line) and inner (green line) optimal perturbations versus time; the dashed line indicates the energy of $\boldsymbol{\nabla} \cdot \boldsymbol{\tau}$.

transient, the energy of this term oscillates around the energy of $\nabla \cdot \tau$, confirming the consistency of the proposed approach.

It is noteworthy that these equations are not suitable for a linear stability analysis; in fact, $\tilde{\boldsymbol{u}}$ cannot be considered infinitesimal, since it contains both the coherent $(\hat{\boldsymbol{u}})$ and fluctuating $\left(\boldsymbol{u}^{\prime}\right)$ part of the disturbances, the latter being non-zero in a turbulent flow. Whereas, previous linear instability approaches such as those used by Pujals et al. (2009), Cossu et al. (2009) and Hwang \& Cossu (2010a) used a triple decomposition approach (see Reynolds \& Hussain 1972) where $\boldsymbol{u}=\boldsymbol{U}+\hat{\boldsymbol{u}}+\boldsymbol{u}^{\prime}$, optimizing only the coherent part of the perturbation. However, the model used in these works cannot be easily extended to a nonlinear framework, since it neglects the long-time average of the nonlinear coherent perturbation term $\overline{\hat{\boldsymbol{u}} \hat{\boldsymbol{u}}}$, an hypothesis that cannot be extended to the case of finite-amplitude perturbations, where this term should be large (see also Viola et al. 2014).

Finally, it is worth pointing out that, using a triple decomposition approach, an equation formally equivalent to (A 3) for the coherent part of the perturbation can be derived under the assumption that the variance of the probability distribution of the fluctuating part of the perturbation is small with respect to the Reynolds stress tensor $\boldsymbol{\tau}$, so that the phase average of the fluctuating nonlinear term $\left\langle\boldsymbol{u}^{\prime} \boldsymbol{u}^{\prime}\right\rangle$ can be neglected.

\section{REFERENCES}

Adrian, R. J. 2007 Hairpin vortex organization in wall turbulence. Phys. Fluids 19 (4), 041301. ADRIAN, R. J., BALACHANDAR, S. \& Lin, Z. C. 2001 Spanwise growth of vortex structure in wall turbulence. KSME Intl J. 15 (12), 1741-1749.

Del Álamo, J. C. \& Jiménez, J. 2003 Spectra of the very large anisotropic scales in turbulent channels. Phys. Fluids 15 (6), L41.

Andersson, P., Brandt, L., Bottaro, A. \& Henningson, D. S. 2001 On the breakdown of boundary layer streaks. J. Fluid Mech. 428, 29-60. 
Barkley, D., Song, B., Mukund, V., Lemoult, G., Avila, M. \& Hof, B. 2015 The rise of fully turbulent flow. Nature 526, 550-553.

Blackburn, H. M., Mansour, N. N. \& Cantwell, B. J. 1996 Topology of fine-scale motions in turbulent channel flow. J. Fluid Mech. 310, 269-292.

Bogard, D. G. \& Tiederman, W. G. 1986 Burst detection with single-point velocity measurements. J. Fluid Mech. 162, 389-413.

Butler, K. M. \& FARRell, B. F. 1993 Optimal perturbations and streak spacing in wall-bounded turbulent shear flow. Phys. Fluids A 5 (3), 774-777.

Chen, J., Hussain, F., PeI, J. \& SHE, Z.-S. 2014 Velocity-vorticity correlation structure in turbulent channel flow. J. Fluid Mech. 742, 291-307.

Cherubini, S. \& De Palma, P. 2013 Nonlinear optimal perturbations in a Couette flow: bursting and transition. J. Fluid Mech. 716, 251-279.

Cherubini, S., De Palma, P. \& Robinet, J.-C. 2015 Nonlinear optimals in the asymptotic suction boundary layer: transition thresholds and symmetry breaking. Phys. Fluids 27 (3), 034108.

Cherubini, S., De Palma, P., Robinet, J.-C. \& Bottaro, A. 2010 Rapid path to transition via nonlinear localized optimal perturbations in a boundary-layer flow. Phys. Rev. E 82 (6), 066302.

Chong, M. S., Perry, A. E. \& CAntwell, B. J. 1990 A general classification of three-dimensional flow fields. Phys. Fluids A 2 (5), 765-777.

Cossu, C., Pujals, G. \& Depardon, S. 2009 Optimal transient growth and very large-scale structures in turbulent boundary layers. J. Fluid Mech. 619, 79-94.

Deville, M. O., Fischer, P. F. \& Mund, E. H. 2002 High-Order Methods for Incompressible Fluid Flow, vol. 9. Cambridge University Press.

Duguet, Y., Monokrousos, A., Brandt, L. \& Henningson, D. S. 2013 Minimal transition thresholds in plane Couette flow. Phys. Fluids 25 (8), 084103.

Eitel-Amor, G., Örlü, R., Schlatter, P. \& Flores, O. 2015 Hairpin vortices in turbulent boundary layers. Phys. Fluids 27 (2), 025108.

Faisst, H. \& Eckhardt, B. 2003 Travelling waves in pipe flow. Phys. Rev. Lett. 91, 224502.

Farano, M., Cherubini, S., Robinet, J.-C. \& De Palma, P. 2015 Hairpin-like optimal perturbations in plane poiseuille flow. J. Fluid Mech. 775, R2.

Farano, M., Cherubini, S., Robinet, J.-C. \& De Palma, P. 2016 Subcritical transition scenarios via linear and nonlinear localized optimal perturbations in plane poiseuille flow. Fluid Dyn. Res. 48, 061409.

Fischer, P. F., Lottes, J. W. \& Kerkemeir, S. G. 2008 Nek5000 Web pages. http://nek5000.mcs.anl.gov.

Flores, O. \& Jimenez, J. 2010 Hierarchy of minimal flow units in the logarithmic layer. Phys. Fluids 22, 071704.

Foures, D. P. G., Caulfield, C. P. \& Schmid, P. J. 2013 Localization of flow structures using $\infty$-norm optimization. J. Fluid Mech. 729, 672-701.

Hamilton, J. M., Kim, J. \& WalefFe, F. 1995 Regeneration mechanisms of near-wall turbulence structures. J. Fluid Mech. 287, 317-348.

Heist, D. K., HanRatTy, T. J. \& NA, Y. 2000 Observations of the formation of streamwise vortices by rotation of arch vortices. Phys. Fluids 12 (11), 2965-2975.

Hof, B., van Doorne, C. W. H., Westerweel, J., Nieuwstadt, F. T. M., Faisst, H., Eckhardt, B., Wedin, H., Kerswell, R. R. \& Waleffe, F. 2004 Experimental observation of nonlinear traveling waves in turbulent pipe flow. Science 305, 1594-1598.

HoyAs, S. \& JimÉnEZ, J. 2006 Scaling of the velocity fluctuations in turbulent channels up to $r e_{\tau}=2003$. Phys. Fluids 18 (1), 011702.

Hwang, J., Lee, J., Sung, H. J. \& ZAKI, T. A. 2016 Inner-outer interactions of large-scale structures in turbulent channel flow. J. Fluid Mech. 790, 128-157.

HWANG, Y. 2015 Statistical structure of self-sustaining attached eddies in turbulent channel flow. J. Fluid Mech. 767, 254-289.

Hwang, Y. \& Bengana, Y. 2016 Self-sustaining process of minimal attached eddies in turbulent channel flow. J. Fluid Mech. 795, 708-738. 
Hwang, Y. \& Cossu, C. 2010a Amplification of coherent streaks in the turbulent Couette flow: an input-output analysis at low Reynolds number. J. Fluid Mech. 643, 333-348.

Hwang, Y. \& Cossu, C. $2010 b$ Self-sustained process at large scales in turbulent channel flow. Phys. Rev. Lett. 105 (4), 044505.

JiméneZ, J. 1999 The physics of wall turbulence. Physica A 263 (1), 252-262.

JiméneZ, J. 2013 How linear is wall-bounded turbulence? Phys. Fluids 25, 110814.

JimÉneZ, J. 2015 Direct detection of linearized bursts in turbulence. Phys. Fluids 27, 065102.

JimÉnez, J. \& MoIn, P. 1991 The minimal flow unit in near-wall turbulence. J. Fluid Mech. 225, 213-240.

Jiménez, J. J., Kawahara, G., Simens, M. P., Nagata, N. \& Shiba, M. 2005 Characterization of near-wall turbulence in terms of equilibrium and bursting solutions. Phys. Fluids 17, 015105.

Jiménez, J. J. \& Pinelli, A. 1999 The autonomous cycle of near-wall turbulence. J. Fluid Mech. 389, 335-359.

Kim, J., Moin, P. \& Moser, R. 1987 Turbulence statistics in fully developed channel flow at low Reynolds number. J. Fluid Mech. 177, 133-166.

Kline, S. J., Reynold, W. C., Schraub, F. \& Rundstander, P. W. 1967 The structure of turbulent boundary layer flows. J. Fluid Mech. 30, 741-773.

LANDAHL, M. T. 1980 A note on an algebraic instability of inviscid parallel shear flows. J. Fluid Mech. 98 (02), 243-251.

Lee, M. \& Moser, R. D. 2015 Direct numerical simulation of turbulent channel flow up to re5200. J. Fluid Mech. 774, 395-415.

Lemoult, G., Shi, L., Avila, K., Jalikop, S. V., Avila, M. \& Hof, B. 2016 Directed percolation phase transition to sustained turbulence in Couette flow. Nat. Phys. 12, 254-258.

Marusic, I., Mathis, R. \& Hutchins, N. 2010 Predictive model for wall-bounded turbulent flow. Science 329, 193-196.

MoIn, P. \& KIM, J. 1985 The structure of the vorticity field in turbulent channel flow. Part 1. Analysis of instantaneous fields and statistical correlations. J. Fluid Mech. 155, 441-464.

Mösta, P., Ott, C. D., Radice, D., Roberts, L. F., Schnetter, E. \& HaAs, R. 2015 A largescale dynamo and magnetoturbulence in rapidly rotating core-collapse supernovae. Nature $\mathbf{5 2 8}$, 376-379.

Moum, J. N., Perlin, A., NAsh, J. D. \& Mcphaden, M. J. 2013 Seasonal sea surface cooling in the equatorial pacific cold tongue controlled by ocean mixing. Nature 500, 64-67.

ORR, W. M'F. 1907 The stability or instability of the steady motions of a perfect liquid and of a viscous liquid. Part ii: a viscous liquid. In Proceedings of the Royal Irish Academy. Section A: Mathematical and Physical Sciences, pp. 69-138. JSTOR.

PAnton, R. L. 2001 Overview of the self-sustaining mechanisms of wall turbulence. Prog. Aerosp. Sci. 37 (4), 341-383.

Pringle, C. C. T., Willis, A. P. \& Kerswell, R. R. 2012 Minimal seeds for shear flow turbulence: using nonlinear transient growth to touch the edge of chaos. J. Fluid Mech. 702, 415-443.

Pujals, G., García-Villalba, M., Cossu, C. \& Depardon, S. 2009 A note on optimal transient growth in turbulent channel flows. Phys. Fluids 21 (1), 015109.

Rabin, S. M. E., Caulfield, C. P. \& Kerswell, R. R. 2012 Triggering turbulence efficiently in plane Couette flow. J. Fluid Mech. 712, 244-272.

Reynolds, W. C. \& Hussain, A. K. M. F. 1972 The mechanics of an organized wave in turbulent shear flow. Part 3. Theoretical models and comparisons with experiments. J. Fluid Mech. 54 (02), 263-288.

Robinson, S. K. 1991 Coherent motions in the turbulent boundary layer. Annu. Rev. Fluid Mech. 23 (1), 601-639.

Sano, M. \& TAmaI, K. 2016 A universal transition to turbulence in channel flow. Nat. Phys. 12, 249-253.

Schmid, P. J. \& Henningson, D. S. 2012 Stability and Transition in Shear Flows, vol. 142. Springer. 
Schoppa, W. \& Hussain, F. 2002 Coherent structure generation in near-wall turbulence. J. Fluid Mech. 453, 57-108.

Tomkins, C. D. \& AdRiAn, R. J. 2003 Spanwise structure and scale growth in turbulent boundary layers. J. Fluid Mech. 490, 37-74.

Townsend, A. A. 1980 The Structure of Turbulent Shear Flow. Cambridge University Press.

Viola, F., Iungo, G. V., Camarri, S., Port-Agel, F. \& Gallaire, F. 2014 Prediction of the hub vortex instability in a wind turbine wake: stability analysis with eddy-viscosity models calibrated on wind tunnel data. J. Fluid Mech. 750, R1.

WALEFFe, F. 1997 On a self-sustaining process in shear flows. Phys. Fluids 9, 883-901.

WALeffe, F. 1998 Three-dimensional coherent states in plane shear flow. Phys. Rev. Lett. 81, 4140-4143.

WANG, Y., HUANG, W. \& XU, C. 2015 On hairpin vortex generation from near-wall streamwise vortices. Acta Mechanica Sin. 31 (2), 139-152.

Zhou, J., Adrian, R. J., Balachandar, S. \& Kendall, T. M. 1999 Mechanisms for generating coherent packets of hairpin vortices in channel flow. J. Fluid Mech. 387, 353-396. 